\title{
On the centre of two-parameter quantum groups
}

\author{
Georgia Benkart
}

Department of Mathematics, University of Wisconsin, Madison, WI 53706, USA (benkart@math.wisc.edu)

\author{
Seok-Jin Kang \\ Department of Mathematical Sciences, Seoul National University, \\ San 56-1 Shinrim-dong, Kwanak-ku, Seoul 151-747, Korea \\ (sjkang@math.snu.ac.kr) \\ Kyu-Hwan Lee \\ Department of Mathematics, University of Connecticut, Storrs, \\ CT 06269, USA (khlee@math.uconn.edu)
}

(MS received 8 April 2004; accepted 28 July 2005)

\begin{abstract}
We describe Poincaré-Birkhoff-Witt bases for the two-parameter quantum groups $U=U_{r, s}\left(\mathfrak{s l}_{n}\right)$ following Kharchenko and show that the positive part of $U$ has the structure of an iterated skew polynomial ring. We define an ad-invariant bilinear form on $U$, which plays an important role in the construction of central elements. We introduce an analogue of the Harish-Chandra homomorphism and use it to determine the centre of $U$.
\end{abstract}

\section{Introduction}

In this paper we determine the centre of the two-parameter quantum groups $U=$ $U_{r, s}\left(\mathfrak{s l}_{n}\right)$, which are the same algebras as those introduced by Takeuchi in [35, $36]$, but with the opposite co-product. As shown in [4,5], these quantum groups are Drinfel'd doubles and have an R-matrix. They are related to the down-up algebras in [2,3] and to the multi-parameter quantum groups of Chin and Musson [8] and Dobrev and Parashar [10]. In the analogous quantum function algebra setting, allowing two parameters unifies the Drinfel'd-Jimbo quantum groups $(r=q, s=$ $\left.q^{-1}\right)$ in [11] with the Dipper-Donkin quantum groups $\left(r=1, s=q^{-1}\right)$ in [9].

For the one-parameter quantum groups $U_{q}(\mathfrak{g})$ corresponding to finite-dimensional simple Lie algebras $\mathfrak{g}$, there is a sizeable literature [7, 15, 21-28, 30-32, 37-39] dealing with Poincaré-Birkhoff-Witt (PBW) bases. For the multi-parameter quantum groups associated with $\mathfrak{g}$ of classical type, Kharchenko [21] constructed PBW bases by first determining Gröbner-Shirshov bases for them. We show in this paper that Kharchenko's results, when applied to the algebra $U=U_{r, s}\left(\mathfrak{s l}_{n}\right)$, yield useful commutation relations, which enable us to prove that the positive part $U^{+}$of $U$ has the structure of an iterated skew polynomial ring. As a consequence of that result, $U^{+}$modulo any prime ideal is a domain. The commutation relations also play an essential role in [6], where finite-dimensional restricted two-parameter quantum

(C) 2006 The Royal Society of Edinburgh 
groups $\mathfrak{u}_{r, s}\left(\mathfrak{g l}_{n}\right)$ and $\mathfrak{u}_{r, s}\left(\mathfrak{s l}_{n}\right)$ are constructed when $r$ and $s$ are roots of unity. These restricted quantum groups are Drinfel'd doubles and are ribbon Hopf algebras under suitable restrictions on $r$ and $s$.

Much work has been done on the centre of quantum groups for finite-dimensional simple Lie algebras $[1,12,19,28,29,34,37]$, and also for (generalized) Kac-Moody (super)algebras $[13,16,20]$. The approach taken in many of these papers (and adopted here as well) is to define a bilinear form on the quantum group which is invariant under the adjoint action. This quantum version of the Killing form is often referred to in the one-parameter setting as the Rosso form (see [34]). The next step involves constructing an analogue $\xi$ of the Harish-Chandra map. It is straightforward to show that the map $\xi$ is an injective algebra homomorphism. The main difficulty lies in determining the image of $\xi$ and in finding enough central elements to prove that the map $\xi$ is surjective. In the two-parameter case, a new phenomenon arises: the $n$ odd and $n$ even cases behave differently. Additional central elements arise when $n$ is even, which complicates the description in that case.

Our paper is organized as follows. In $\S 2$, we briefly recall the definition and basic properties of the two-parameter quantum group $U=U_{r, s}\left(\mathfrak{s l}_{n}\right)$. In $\S 3$, we describe the commutation relations which determine a Gröbner-Shirshov basis and allow a PBW basis to be constructed, and we prove that the positive part of $U$ has an iterated skew polynomial ring structure. The next section is devoted to the construction of a bilinear form and the proof of its invariance under the adjoint action. In the final section, we define a Harish-Chandra homomorphism $\xi$ and determine the centre of $U$ by specifying the image of $\xi$ and constructing central elements explicitly.

\section{Two-parameter quantum groups}

Let $\mathbb{K}$ be an algebraically closed field of characteristic 0 . Assume that $\Phi$ is a finite root system of type $A_{n-1}$ with $\Pi$ a base of simple roots. We regard $\Phi$ as a subset of a Euclidean space $\mathbb{R}^{n}$ with an inner product $\langle\cdot, \cdot\rangle$. We let $\epsilon_{1}, \ldots, \epsilon_{n}$ denote an orthonormal basis of $\mathbb{R}^{n}$, and suppose that $\Pi=\left\{\alpha_{j}=\epsilon_{j}-\epsilon_{j+1} \mid j=1, \ldots, n-1\right\}$ and that $\Phi=\left\{\epsilon_{i}-\epsilon_{j} \mid 1 \leqslant i \neq j \leqslant n\right\}$.

Fix non-zero elements $r, s$ in the field $\mathbb{K}$. Here we assume $r \neq s$. Let $\tilde{U}=U_{r, s}\left(\mathfrak{g l}_{n}\right)$ be the unital associative algebra over $\mathbb{K}$ generated by elements $e_{j}, f_{j}(1 \leqslant j<n)$, and $a_{i}^{ \pm 1}, b_{i}^{ \pm 1}(1 \leqslant i \leqslant n)$, which satisfy the following relations:

(R1) the $a_{i}^{ \pm 1}, b_{j}^{ \pm 1}$ all commute with one another and $a_{i} a_{i}^{-1}=b_{j} b_{j}^{-1}=1$;

(R2) $a_{i} e_{j}=r^{\left\langle\epsilon_{i}, \alpha_{j}\right\rangle} e_{j} a_{i}$ and $a_{i} f_{j}=r^{-\left\langle\epsilon_{i}, \alpha_{j}\right\rangle} f_{j} a_{i}$;

(R3) $b_{i} e_{j}=s^{\left\langle\epsilon_{i}, \alpha_{j}\right\rangle} e_{j} b_{i}$ and $b_{i} f_{j}=s^{-\left\langle\epsilon_{i}, \alpha_{j}\right\rangle} f_{j} b_{i}$;

$$
\left[e_{i}, f_{j}\right]=\frac{\delta_{i, j}}{r-s}\left(a_{i} b_{i+1}-a_{i+1} b_{i}\right)
$$

$\left[e_{i}, e_{j}\right]=\left[f_{i}, f_{j}\right]=0$ if $|i-j|>1$

$$
\begin{array}{r}
e_{i}^{2} e_{i+1}-(r+s) e_{i} e_{i+1} e_{i}+r s e_{i+1} e_{i}^{2}=0 \\
e_{i} e_{i+1}^{2}-(r+s) e_{i+1} e_{i} e_{i+1}+r s e_{i+1}^{2} e_{i}=0
\end{array}
$$




$$
\begin{array}{r}
f_{i}^{2} f_{i+1}-\left(r^{-1}+s^{-1}\right) f_{i} f_{i+1} f_{i}+r^{-1} s^{-1} f_{i+1} f_{i}^{2}=0 \\
f_{i} f_{i+1}^{2}-\left(r^{-1}+s^{-1}\right) f_{i+1} f_{i} f_{i+1}+r^{-1} s^{-1} f_{i+1}^{2} f_{i}=0 .
\end{array}
$$

Let $U=U_{r, s}\left(\mathfrak{s l}_{\mathfrak{n}}\right)$ be the subalgebra of $\tilde{U}=U_{r, s}\left(\mathfrak{g l}_{\mathfrak{n}}\right)$ generated by the elements $e_{j}, f_{j}, \omega_{j}^{ \pm 1}$ and $\left(\omega_{j}^{\prime}\right)^{ \pm 1}(1 \leqslant j<n)$, where

$$
\omega_{j}=a_{j} b_{j+1} \quad \text { and } \quad \omega_{j}^{\prime}=a_{j+1} b_{j} .
$$

These elements satisfy (R5)-(R7) along with the following relations:

$\left(\mathrm{R} 1^{\prime}\right)$ the $\omega_{i}^{ \pm 1},\left(\omega_{j}^{\prime}\right)^{ \pm 1}$ all commute with one another and $\omega_{i} \omega_{i}^{-1}=\omega_{j}^{\prime}\left(\omega_{j}^{\prime}\right)^{-1}=1$;

$\left(\mathrm{R} 2^{\prime}\right) \omega_{i} e_{j}=r^{\left\langle\epsilon_{i}, \alpha_{j}\right\rangle} s^{\left\langle\epsilon_{i+1}, \alpha_{j}\right\rangle} e_{j} \omega_{i}$ and $\omega_{i} f_{j}=r^{-\left\langle\epsilon_{i}, \alpha_{j}\right\rangle} s^{-\left\langle\epsilon_{i+1}, \alpha_{j}\right\rangle} f_{j} \omega_{i}$;

$\left(\mathrm{R} 3^{\prime}\right) \omega_{i}^{\prime} e_{j}=r^{\left\langle\epsilon_{i+1}, \alpha_{j}\right\rangle} s^{\left\langle\epsilon_{i}, \alpha_{j}\right\rangle} e_{j} \omega_{i}^{\prime}$ and $\omega_{i}^{\prime} f_{j}=r^{-\left\langle\epsilon_{i+1}, \alpha_{j}\right\rangle} s^{-\left\langle\epsilon_{i}, \alpha_{j}\right\rangle} f_{j} \omega_{i}^{\prime}$;

$\left(\mathrm{R} 4^{\prime}\right)\left[e_{i}, f_{j}\right]=\frac{\delta_{i, j}}{r-s}\left(\omega_{i}-\omega_{i}^{\prime}\right)$.

Let $U^{+}$and $U^{-}$be the subalgebras generated by the elements $e_{i}$ and $f_{i}$, respectively, and let $\tilde{U}^{0}$ and $U^{0}$ be the subalgebras generated by the elements $a_{i}^{ \pm 1}, b_{i}^{ \pm 1}$, $1 \leqslant i \leqslant n$ and $\omega_{i}^{ \pm 1},\left(\omega_{i}^{\prime}\right)^{ \pm 1}, 1 \leqslant i<n$, respectively. It now follows from the defining relations that $\tilde{U}$ has a triangular decomposition: $\tilde{U}=U^{-} \tilde{U}^{0} U^{+}$. Similarly, we have $U=U^{-} U^{0} U^{+}$.

The algebras $\tilde{U}$ and $U$ are Hopf algebras, where the $a_{i}^{ \pm}, b_{i}^{ \pm}$are group-like elements, and the remaining co-products are determined by

$$
\Delta\left(e_{i}\right)=e_{i} \otimes 1+\omega_{i} \otimes e_{i}, \quad \Delta\left(f_{i}\right)=1 \otimes f_{i}+f_{i} \otimes \omega_{i}^{\prime} .
$$

This forces the co-unit and antipode maps to be

$$
\begin{array}{lll}
\varepsilon\left(a_{i}\right)=\varepsilon\left(b_{i}\right)=1, & S\left(a_{i}\right)=a_{i}^{-1}, & S\left(b_{i}\right)=b_{i}^{-1}, \\
\varepsilon\left(e_{i}\right)=\varepsilon\left(f_{i}\right)=0, & S\left(e_{i}\right)=-\omega_{i}^{-1} e_{i}, & S\left(f_{i}\right)=-f_{i}\left(\omega_{i}^{\prime}\right)^{-1} .
\end{array}
$$

Let $Q=\mathbb{Z} \Phi$ denote the root lattice and set $Q^{+}=\bigoplus_{i=1}^{n-1} \mathbb{Z}_{\geqslant 0} \alpha_{i}$. Then, for any $\zeta=\sum_{i=1}^{n-1} \zeta_{i} \alpha_{i} \in Q$, we adopt the shorthand

$$
\omega_{\zeta}=\omega_{1}^{\zeta_{1}} \cdots \omega_{n-1}^{\zeta_{n-1}}, \quad \omega_{\zeta}^{\prime}=\left(\omega_{1}^{\prime}\right)^{\zeta_{1}} \cdots\left(\omega_{n-1}^{\prime}\right)^{\zeta_{n-1}} .
$$

Lemma 2.1 (Benkart and Witherspoon [4, lemma 1.3]). Suppose that

$$
\zeta=\sum_{i=1}^{n-1} \zeta_{i} \alpha_{i} \in Q .
$$

Then

$$
\begin{array}{ll}
\omega_{\zeta} e_{i}=r^{-\left\langle\epsilon_{i+1}, \zeta\right\rangle} s^{-\left\langle\epsilon_{i}, \zeta\right\rangle} e_{i} \omega_{\zeta}, & \omega_{\zeta} f_{i}=r^{\left\langle\epsilon_{i+1}, \zeta\right\rangle} s^{\left\langle\epsilon_{i}, \zeta\right\rangle} f_{i} \omega_{\zeta}, \\
\omega_{\zeta}^{\prime} e_{i}=r^{-\left\langle\epsilon_{i}, \zeta\right\rangle} s^{-\left\langle\epsilon_{i+1}, \zeta\right\rangle} e_{i} \omega_{\zeta}^{\prime}, & \omega_{\zeta}^{\prime} f_{i}=r^{\left\langle\epsilon_{i}, \zeta\right\rangle} s^{\left\langle\epsilon_{i+1}, \zeta\right\rangle} f_{i} \omega_{\zeta}^{\prime} .
\end{array}
$$


There is a grading on $U$ with the degrees of the generators given by

$$
\operatorname{deg} e_{i}=\alpha_{i}, \quad \operatorname{deg} f_{i}=-\alpha_{i}, \quad \operatorname{deg} \omega_{i}=\operatorname{deg} \omega_{i}^{\prime}=0 .
$$

Then, since the defining relations are homogeneous under this grading, the algebra $U$ has a $Q$-grading:

$$
U=\bigoplus_{\zeta \in Q} U_{\zeta}
$$

We also have

$$
U^{+}=\bigoplus_{\zeta \in Q^{+}} U_{\zeta}^{+} \text {and } U^{-}=\bigoplus_{\zeta \in Q^{+}} U_{-\zeta}^{-},
$$

where $U_{\zeta}^{+}=U^{+} \cap U_{\zeta}$ and $U_{-\zeta}^{-}=U^{-} \cap U_{-\zeta}$.

Let $\Lambda=\bigoplus_{i=1}^{n} \mathbb{Z} \epsilon_{i}$ be the weight lattice of $\mathfrak{g l}_{n}$. Corresponding to any $\lambda \in \Lambda$ is an algebra homomorphism $\varrho^{\lambda}: \tilde{U}^{0} \rightarrow \mathbb{K}$ given by

$$
\varrho^{\lambda}\left(a_{i}\right)=r^{\left\langle\epsilon_{i}, \lambda\right\rangle} \text { and } \varrho^{\lambda}\left(b_{i}\right)=s^{\left\langle\epsilon_{i}, \lambda\right\rangle} .
$$

For any $\lambda=\sum_{i=1}^{n} \lambda_{i} \epsilon_{i} \in \Lambda$, we write

$$
a_{\lambda}=a_{1}^{\lambda_{1}} \cdots a_{n}^{\lambda_{n}} \quad \text { and } \quad b_{\lambda}=b_{1}^{\lambda_{1}} \cdots b_{n}^{\lambda_{n}} .
$$

Let $\Lambda_{\mathfrak{s l}}=\bigoplus_{i=1}^{n-1} \mathbb{Z} \varpi_{i}$ be the weight lattice of $\mathfrak{s l}_{n}$, where $\varpi_{i}$ is the fundamental weight

$$
\varpi_{i}=\epsilon_{1}+\cdots+\epsilon_{i}-\frac{i}{n} \sum_{j=1}^{n} \epsilon_{j}
$$

and let

$$
\Lambda_{\mathfrak{s l}}^{+}=\left\{\lambda \in \Lambda_{\mathfrak{s l}} \mid\left\langle\alpha_{i}, \lambda\right\rangle \geqslant 0 \text { for } 1 \leqslant i<n\right\}=\left\{\sum_{i=1}^{n-1} l_{i} \varpi_{i} \mid l_{i} \in \mathbb{Z}_{\geqslant 0}\right\}
$$

denote the set of dominant weights for $\mathfrak{s l}_{n}$. We fix the $n$th $\operatorname{roots} r^{1 / n}$ and $s^{1 / n}$ of $r$ and $s$, respectively, and define, for any $\lambda \in \Lambda_{\mathfrak{s l}}$, an algebra homomorphism $\varrho^{\lambda}: U^{0} \rightarrow \mathbb{K}$ by

$$
\varrho^{\lambda}\left(\omega_{j}\right)=r^{\left\langle\epsilon_{j}, \lambda\right\rangle} s^{\left\langle\epsilon_{j+1}, \lambda\right\rangle} \text { and } \varrho^{\lambda}\left(\omega_{j}^{\prime}\right)=r^{\left\langle\epsilon_{j+1}, \lambda\right\rangle} s^{\left\langle\epsilon_{j}, \lambda\right\rangle} .
$$

In particular, if $\lambda$ belongs to $\Lambda$, then the definition of $\varrho^{\lambda}\left(\omega_{j}\right)$ and $\varrho^{\lambda}\left(\omega_{j}^{\prime}\right)$ coming from (2.2) coincides with (2.4).

Associated with any algebra homomorphism $\psi: U^{0} \rightarrow \mathbb{K}$ is the Verma module $M(\psi)$ with highest weight $\psi$ and its unique irreducible quotient $L(\psi)$. When the highest weight is given by the homomorphism $\varrho^{\lambda}$ for $\lambda \in \Lambda_{\mathfrak{s l}}$, we simply write $M(\lambda)$ and $L(\lambda)$ instead of $M\left(\varrho^{\lambda}\right)$ and $L\left(\varrho^{\lambda}\right)$.

LEMMA 2.2 (Benkart and Witherspoon [5]). We assume that $\mathrm{rs}^{-1}$ is not a root of unity, and let $v_{\lambda}$ be a highest weight vector of $M(\lambda)$ for $\lambda \in \Lambda_{\mathfrak{s l}}^{+}$. The irreducible module $L(\lambda)$ is then given by

$$
L(\lambda)=M(\lambda) /\left(\sum_{i=1}^{n-1} U f_{i}^{\left\langle\lambda, \alpha_{i}\right\rangle+1} v_{\lambda}\right)
$$


Let $W$ be the Weyl group of the root system $\Phi$, and let $\sigma_{i} \in W$ denote the reflection corresponding to $\alpha_{i}$ for each $1 \leqslant i<n$. Thus,

$$
\sigma_{i}(\lambda)=\lambda-\left\langle\lambda, \alpha_{i}\right\rangle \alpha_{i} \quad \text { for } \lambda \in \Lambda,
$$

and $\sigma_{i}$ also acts on $\Lambda_{\mathfrak{s l}}$, according to the same formula.

Let $M$ be a finite-dimensional $U$-module on which $U^{0}$ acts semi-simply. Then

$$
M=\bigoplus_{\chi} M_{\chi}
$$

where each $\chi: U^{0} \rightarrow \mathbb{K}$ is an algebra homomorphism, and

$$
M_{\chi}=\left\{m \in M \mid \omega_{i} m=\chi\left(\omega_{i}\right) m \text { and } \omega_{i}^{\prime} m=\chi\left(\omega_{i}^{\prime}\right) m \text { for all } i\right\} .
$$

For brevity we write $M_{\lambda}$ for the weight space $M_{\varrho^{\lambda}}$ for $\lambda \in \Lambda_{\mathfrak{s} I}$.

Proposition 2.3. Assume that $r^{-1}$ is not a root of unity and that $\lambda \in \Lambda_{\mathfrak{s l}}^{+}$. Then

$$
\operatorname{dim} L(\lambda)_{\mu}=\operatorname{dim} L(\lambda)_{\sigma(\mu)}
$$

for all $\mu \in \Lambda_{\mathfrak{s l}}$ and $\sigma \in W$.

Proof. This is an immediate consequence of [5, proposition 2.8 and the proof of lemma 2.12].

\section{PBW-type bases}

From now on we assume that $r+s \neq 0$ (or equivalently, $r^{-1}+s^{-1} \neq 0$ ), and the ordering $(k, l)<(i, j)$ always means relative to the lexicographic ordering.

We define inductively

$$
\mathcal{E}_{j, j}=e_{j} \quad \text { and } \quad \mathcal{E}_{i, j}=e_{i} \mathcal{E}_{i-1, j}-r^{-1} \mathcal{E}_{i-1, j} e_{i}, \quad i>j .
$$

The defining relations for $U^{+}$in (R6) can be reformulated as saying

$$
\begin{aligned}
\mathcal{E}_{i+1, i} e_{i} & =s^{-1} e_{i} \mathcal{E}_{i+1, i}, \\
e_{i+1} \mathcal{E}_{i+1, i} & =s^{-1} \mathcal{E}_{i+1, i} e_{i+1} .
\end{aligned}
$$

Even though the relations in the following theorem can be deduced from [21, theorem $A_{n}$ ], we include a self-contained proof in the appendix for the convenience of the reader.

Theorem 3.1 (Kharchenko [21]). Assume that $(i, j)>(k, l)$ in the lexicographic order. Then the following relations hold in the algebra $U^{+}$:

(1) $\mathcal{E}_{i, j} \mathcal{E}_{k, l}-r^{-1} \mathcal{E}_{k, l} \mathcal{E}_{i, j}-\mathcal{E}_{i, l}=0$ if $j=k+1$;

(2) $\mathcal{E}_{i, j} \mathcal{E}_{k, l}-\mathcal{E}_{k, l} \mathcal{E}_{i, j}=0$ if $i>k \geqslant l>j$ or $j>k+1$;

(3) $\mathcal{E}_{i, j} \mathcal{E}_{k, l}-s^{-1} \mathcal{E}_{k, l} \mathcal{E}_{i, j}=0$ if $i=k \geqslant j>l$ or $i>k \geqslant j=l$;

(4) $\mathcal{E}_{i, j} \mathcal{E}_{k, l}-r^{-1} s^{-1} \mathcal{E}_{k, l} \mathcal{E}_{i, j}+\left(r^{-1}-s^{-1}\right) \mathcal{E}_{k, j} \mathcal{E}_{i, l}=0$ if $i>k \geqslant j>l$. 
Let $E=\left\{e_{1}, e_{2}, \ldots, e_{n-1}\right\}$ be the set of generators of the algebra $U^{+}$. We introduce a linear ordering $\prec$ on $E$ by saying $e_{i} \prec e_{j}$ if and only if $i<j$. We extend this ordering to the set of monomials in $E$ so that it becomes the degree-lexicographic ordering; that is, for $u=u_{1} u_{2} \cdots u_{p}$ and $v=v_{1} v_{2} \cdots v_{q}$ with $u_{i}, v_{j} \in E$, we have $u \prec v$ if and only if $p<q$ or $p=q$ and $u_{i} \prec v_{i}$ for the first $i$ such that $u_{i} \neq v_{i}$. Let $\mathcal{A}_{E}$ be the free associative algebra generated by $E$ and $\mathcal{S} \subset \mathcal{A}_{E}$ be the set consisting of the following elements:

$$
\begin{aligned}
\mathcal{E}_{i, j} \mathcal{E}_{k, l}-\mathcal{E}_{k, l} \mathcal{E}_{i, j} & \text { if } i>k \geqslant l>j \text { or } j>k+1, \\
\mathcal{E}_{i, j} \mathcal{E}_{k, l}-s^{-1} \mathcal{E}_{k, l} \mathcal{E}_{i, j} & \text { if } i=k \geqslant j>l \text { or } i>k \geqslant j=l, \\
\mathcal{E}_{i, j} \mathcal{E}_{k, l}-r^{-1} s^{-1} \mathcal{E}_{k, l} \mathcal{E}_{i, j}+\left(r^{-1}-s^{-1}\right) \mathcal{E}_{k, j} \mathcal{E}_{i, l} & \text { if } i>k \geqslant j>l .
\end{aligned}
$$

The elements of $\mathcal{S}$ just correspond to relations (2)-(4) of theorem 3.1. Note that we may take $\mathcal{S}$ to be the set of defining relations for the algebra $U^{+}$, since $\mathcal{S}$ contains all the (original) defining relations (R5) and (R6) of $U^{+}$, and the other relations in $\mathcal{S}$ are all consequences of (R5) and (R6).

The following theorem is a special case of in [21, theorem $\left.\boldsymbol{A}_{n}\right]$ and its consequences. Also, one can prove it using an argument similar to that in $[7]$ or $[39,40]$.

Theorem 3.2 (Kharchenko [21]). Assume that $r, s \in \mathbb{K}^{\times}$and $r+s \neq 0$. Then

(i) the set $\mathcal{S}$ is a Gröbner-Shirshov basis for the algebra $U^{+}$with respect to the degree-lexicographic ordering,

(ii) $\mathcal{B}_{0}=\left\{\mathcal{E}_{i_{1}, j_{1}} \mathcal{E}_{i_{2}, j_{2}} \cdots \mathcal{E}_{i_{p}, j_{p}} \mid\left(i_{1}, j_{1}\right) \leqslant\left(i_{2}, j_{2}\right) \leqslant \cdots \leqslant\left(i_{p}, j_{p}\right)\right\}$ (lexicographical ordering) is a linear basis of the algebra $U^{+}$,

(iii) $\mathcal{B}_{1}=\left\{e_{i_{1}, j_{1}} e_{i_{2}, j_{2}} \cdots e_{i_{p}, j_{p}} \mid\left(i_{1}, j_{1}\right) \leqslant\left(i_{2}, j_{2}\right) \leqslant \cdots \leqslant\left(i_{p}, j_{p}\right)\right\}$ (lexicographical ordering) is a linear basis of the algebra $U^{+}$, where $e_{i, j}=e_{i} e_{i-1} \cdots e_{j}$ for $i \geqslant j$.

REMARK 3.3. If we define $\mathcal{F}_{i, j}$ inductively by

$$
\mathcal{F}_{j, j}=f_{j} \quad \text { and } \quad \mathcal{F}_{i, j}=f_{i} \mathcal{F}_{i-1, j}-s \mathcal{F}_{i-1, j} f_{i}, \quad i>j,
$$

and denote by $f_{i, j}$ the monomial $f_{i, j}=f_{i} f_{i-1} \cdots f_{j}, i \geqslant j$, then we have linear bases for the algebra $U^{-}$as in theorem 3.2. Note that $\tilde{U}^{0}$ and $U^{0}$, which are group algebras, have obvious linear bases. Combining these bases using the triangular decomposition $\tilde{U}=U^{-} \tilde{U}^{0} U^{+}$and $U=U^{-} U^{0} U^{+}$, we obtain PBW bases for the entire algebras $\tilde{U}$ and $U$, respectively.

Now we turn our attention to showing that the algebra $U^{+}$is an iterated skew polynomial ring over $\mathbb{K}$ and that any prime ideal $P$ of $U^{+}$is completely prime (that is, $U^{+} / P$ is a domain) when $r$ and $s$ are 'generic' (see proposition 3.6 for the precise statement). Our approach is similar to that in [33], which treats the one-parameter quantum group case. Recall that if $\varphi$ is an automorphism of an algebra $R$, then $\vartheta \in \operatorname{End}(R)$ is a $\varphi$-derivation if $\vartheta(a b)=\vartheta(a) b+\varphi(a) \vartheta(b)$ for all $a, b \in R$. The skew polynomial ring $R[x ; \varphi, \vartheta]$ consists of polynomials $\sum_{i} a_{i} x^{i}$ over $R$, where $x a=\varphi(a) x+\vartheta(a)$ for all $a \in R$. 
For each $(i, j), 1 \leqslant j \leqslant i<n$, we define an algebra automorphism $\varphi_{i, j}$ of $U$ by

$$
\varphi_{i, j}(u)=\omega_{\alpha_{i}+\cdots+\alpha_{j}} u \omega_{\alpha_{i}+\cdots+\alpha_{j}}^{-1} \text { for all } u \in U .
$$

Using lemma 2.1, one can check that if $(k, l)<(i, j)$, then

$$
\varphi_{i, j}\left(\mathcal{E}_{k, l}\right)= \begin{cases}r^{-1} \mathcal{E}_{k, l} & \text { if } j=k+1, \\ \mathcal{E}_{k, l} & \text { if } i>k \geqslant l>j \text { or } j>k+1, \\ s^{-1} \mathcal{E}_{k, l} & \text { if } i=k \geqslant j>l \text { or } i>k \geqslant j=l, \\ r^{-1} s^{-1} \mathcal{E}_{k, l} & \text { if } i>k \geqslant j>l .\end{cases}
$$

Hence, the automorphism $\varphi_{i, j}$ preserves the subalgebra $U_{i, j}^{+}$of $U^{+}$generated by the vectors $\mathcal{E}_{k, l}$ for $(k, l)<(i, j)$. We denote the induced automorphism of $U_{i, j}^{+}$by the same symbol $\varphi_{i, j}$.

Now we define a $\varphi_{i, j}$-derivation $\vartheta_{i, j}$ on $U_{i, j}^{+}$by

$$
\vartheta_{i, j}\left(\mathcal{E}_{k, l}\right)=\mathcal{E}_{i, j} \mathcal{E}_{k, l}-\varphi_{i, j}\left(\mathcal{E}_{k, l}\right) \mathcal{E}_{i, j}= \begin{cases}\mathcal{E}_{i, l}, & j=k+1, \\ \left(r^{-1}-s^{-1}\right) \mathcal{E}_{k, j} \mathcal{E}_{i, l}, & i>k \geqslant j>l, \\ 0 & \text { otherwise }\end{cases}
$$

It is easy to see that $\vartheta_{i, j}$ is indeed a $\varphi_{i, j}$-derivation (cf. [33, lemma 3, p. 62]). With $\varphi_{i, j}$ and $\vartheta_{i, j}$ at hand, the next proposition follows immediately.

Proposition 3.4. The algebra $U^{+}$is an iterated skew polynomial ring whose structure is given by

$$
U^{+}=\mathbb{K}\left[\mathcal{E}_{1,1}\right]\left[\mathcal{E}_{2,1} ; \varphi_{2,1}, \vartheta_{2,1}\right] \cdots\left[\mathcal{E}_{n-1, n-1} ; \varphi_{n-1, n-1}, \vartheta_{n-1, n-1}\right] .
$$

Proof. Note that all the relations in theorem 3.1 can be condensed into a single expression:

$$
\mathcal{E}_{i, j} \mathcal{E}_{k, l}=\varphi_{i, j}\left(\mathcal{E}_{k, l}\right) \mathcal{E}_{i, j}+\vartheta_{i, j}\left(\mathcal{E}_{k, l}\right), \quad(i, j)>(k, l) .
$$

The proposition then easily follows from theorem 3.2.

The other result of this section requires an additional lemma.

LEMma 3.5. The automorphism $\varphi_{i, j}$ and the $\varphi_{i, j}$-derivation $\vartheta_{i, j}$ of $U_{i, j}^{+}$satisfy

$$
\varphi_{i, j} \vartheta_{i, j}=r s^{-1} \vartheta_{i, j} \varphi_{i, j}
$$

Proof. For $(k, l)<(i, j)$, the definitions imply that

$$
\left(\varphi_{i, j} \vartheta_{i, j}\right)\left(\mathcal{E}_{k, l}\right)= \begin{cases}s^{-1} \mathcal{E}_{i, l} & \text { if } j=k+1 \\ \left(r^{-1}-s^{-1}\right) s^{-2} \mathcal{E}_{k, j} \mathcal{E}_{i, l} & \text { if } i>k \geqslant j>l \\ 0 & \text { otherwise }\end{cases}
$$

On the other hand, for $(k, l)<(i, j)$,

$$
\left(\vartheta_{i, j} \varphi_{i, j}\right)\left(\mathcal{E}_{k, l}\right)= \begin{cases}r^{-1} \mathcal{E}_{i, l} & \text { if } j=k+1 \\ \left(r^{-1}-s^{-1}\right) r^{-1} s^{-1} \mathcal{E}_{k, j} \mathcal{E}_{i, l} & \text { if } i>k \geqslant j>l, \\ 0 & \text { otherwise }\end{cases}
$$

Comparing these two calculations, we arrive at the result. 
We now obtain the following proposition.

Proposition 3.6. Assume that the subgroup of $\mathbb{K}^{\times}$generated by $r$ and $s$ is torsionfree. Then all prime ideals of $U^{+}$are completely prime.

Proof. The proof follows directly from proposition 3.4, lemma 3.5 and [14, theorem 2.3].

\section{An invariant bilinear form on $U$}

Assume that $B$ is the subalgebra of $U$ generated by $e_{j}, \omega_{j}^{ \pm 1}, 1 \leqslant j<n$, and $B^{\prime}$ is the subalgebra of $U$ generated by $f_{j},\left(\omega_{j}^{\prime}\right)^{ \pm 1}, 1 \leqslant j<n$. We recall some results in [4].

Proposition 4.1 (Benkart and Witherspoon [4, lemma 2.2]). There is a Hopf pairing $(\cdot, \cdot)$ on $B^{\prime} \times B$ such that, for $x_{1}, x_{2} \in B, y_{1}, y_{2} \in B^{\prime}$, the following properties hold:

(i) $\left(1, x_{1}\right)=\varepsilon\left(x_{1}\right),\left(y_{1}, 1\right)=\varepsilon\left(y_{1}\right)$;

(ii) $\left(y_{1}, x_{1} x_{2}\right)=\left(\Delta^{\mathrm{op}}\left(y_{1}\right), x_{1} \otimes x_{2}\right),\left(y_{1} y_{2}, x_{1}\right)=\left(y_{1} \otimes y_{2}, \Delta\left(x_{1}\right)\right)$;

(iii) $\left(S^{-1}\left(y_{1}\right), x_{1}\right)=\left(y_{1}, S\left(x_{1}\right)\right)$;

(iv) $\left(f_{i}, e_{j}\right)=\frac{\delta_{i, j}}{s-r}$;

$$
\begin{aligned}
\left(\omega_{i}^{\prime}, \omega_{j}\right) & =\left(\omega_{i}^{\prime-1}, \omega_{j}^{-1}\right)=r^{\left\langle\epsilon_{j}, \alpha_{i}\right\rangle} s^{\left\langle\epsilon_{j+1}, \alpha_{i}\right\rangle}=r^{-\left\langle\epsilon_{i+1}, \alpha_{j}\right\rangle} s^{-\left\langle\epsilon_{i}, \alpha_{j}\right\rangle}, \\
\left(\omega_{i}^{\prime-1}, \omega_{j}\right) & =\left(\omega_{i}^{\prime}, \omega_{j}^{-1}\right)=r^{-\left\langle\epsilon_{j}, \alpha_{i}\right\rangle} s^{-\left\langle\epsilon_{j+1}, \alpha_{i}\right\rangle}=r^{\left\langle\epsilon_{i+1}, \alpha_{j}\right\rangle} s^{\left\langle\epsilon_{i}, \alpha_{j}\right\rangle} .
\end{aligned}
$$

It is easy to prove for $\lambda \in Q$ that

$$
\varrho^{\lambda}\left(\omega_{\mu}^{\prime}\right)=\left(\omega_{\mu}^{\prime}, \omega_{-\lambda}\right) \text { and } \varrho^{\lambda}\left(\omega_{\mu}\right)=\left(\omega_{\lambda}^{\prime}, \omega_{\mu}\right) .
$$

From the definition of the co-product, it is apparent that

$$
\Delta(x) \in \bigoplus_{0 \leqslant \nu \leqslant \mu} U_{\mu-\nu}^{+} \omega_{\nu} \otimes U_{\nu}^{+} \quad \text { for any } x \in U_{\mu}^{+},
$$

where ' $\leqslant$ ' is the usual partial order on $Q: \nu \leqslant \mu$ if $\mu-\nu \in Q^{+}$. Thus, for each $i$, $1 \leqslant i<n$, there are elements $p_{i}(x)$ and $p_{i}^{\prime}(x)$ in $U_{\mu-\alpha_{i}}^{+}$such that the component of $\Delta(x)$ in $U_{\mu-\alpha_{i}}^{+} \omega_{i} \otimes U_{\alpha_{i}}^{+}$is equal to $p_{i}(x) \omega_{i} \otimes e_{i}$, and the component of $\Delta(x)$ in $U_{\alpha_{i}}^{+} \omega_{\mu-\alpha_{i}} \otimes U_{\mu-\alpha_{i}}^{+}$is equal to $e_{i} \omega_{\mu-\alpha_{i}} \otimes p_{i}^{\prime}(x)$. Therefore, for $x \in U_{\mu}^{+}$, we can write

$$
\begin{aligned}
\Delta(x) & =x \otimes 1+\sum_{i=1}^{n-1} p_{i}(x) \omega_{i} \otimes e_{i}+\varsigma_{1} \\
& =\omega_{\mu} \otimes x+\sum_{i=1}^{n-1} e_{i} \omega_{\mu-\alpha_{i}} \otimes p_{i}^{\prime}(x)+\varsigma_{2},
\end{aligned}
$$

where $\varsigma_{1}$ and $\varsigma_{2}$ are the sums of terms involving products of more than one $e_{j}$ in the second factor and in the first factor, respectively. 
Lemma 4.2 (Benkart and Witherspoon [4, lemma 4.6]). For all $x \in U_{\zeta}^{+}$and all $y \in U^{-}$, the following hold:

(i) $\left(f_{i} y, x\right)=\left(f_{i}, e_{i}\right)\left(y, p_{i}^{\prime}(x)\right)=(s-r)^{-1}\left(y, p_{i}^{\prime}(x)\right)$;

(ii) $\left(y f_{i}, x\right)=\left(f_{i}, e_{i}\right)\left(y, p_{i}(x)\right)=(s-r)^{-1}\left(y, p_{i}(x)\right)$;

(iii) $f_{i} x-x f_{i}=(s-r)^{-1}\left(p_{i}(x) \omega_{i}-\omega_{i}^{\prime} p_{i}^{\prime}(x)\right)$.

Corollary 4.3. If $\zeta, \zeta^{\prime} \in Q^{+}$with $\zeta \neq \zeta^{\prime}$, then $(y, x)=0$ for all $x \in U_{\zeta}^{+}$and $y \in U_{-\zeta^{\prime}}^{-}$.

Lemma 4.4. Assume that $r s^{-1}$ is not a root of unity and $\zeta \in Q^{+}$is non-zero.

(a) If $y \in U_{-\zeta}^{-}$and $\left[e_{i}, y\right]=0$ for all $i$, then $y=0$.

(b) If $x \in U_{\zeta}^{+}$and $\left[f_{i}, x\right]=0$ for all $i$, then $x=0$.

Proof. Assume that $y \in U_{-\zeta}^{-}$and that $\left[e_{i}, y\right]=0$ holds for all $i$. From the definition of $M(\lambda)$ and lemma 2.2, we can find a sufficiently large $\lambda \in \Lambda_{\mathfrak{s l}}^{+}$such that the map

$$
U_{-\zeta}^{-} \hookrightarrow L(\lambda), \quad u \mapsto u v_{\lambda}
$$

is injective, where $v_{\lambda}$ is a highest weight vector of $L(\lambda)$. Then

$$
U y v_{\lambda}=U^{-} U^{0} U^{+} y v_{\lambda}=U^{-} y U^{0} U^{+} v_{\lambda}=U^{-} y v_{\lambda} \subsetneq L(\lambda)
$$

so that $U y v_{\lambda}$ is a proper submodule of $L(\lambda)$, which must be 0 by the irreducibility of $L(\lambda)$. Thus, $y v_{\lambda}=0$ and $y=0$ by the injectivity of the map above. We can now apply the anti-automorphism $\tau$ of $U$ defined by

$$
\tau\left(e_{i}\right)=f_{i}, \quad \tau\left(f_{i}\right)=e_{i}, \quad \tau\left(\omega_{i}\right)=\omega_{i} \quad \text { and } \quad \tau\left(\omega_{i}^{\prime}\right)=\omega_{i}^{\prime},
$$

to obtain the second assertion.

Lemma 4.5. Assume that $r^{-1}$ is not a root of unity. For $\zeta \in Q^{+}$, the spaces $U_{\zeta}^{+}$ and $U_{-\zeta}^{-}$are non-degenerately paired.

Proof. We use induction on $\zeta$ with respect to the partial order $\leqslant$ on $Q$. The claim holds for $\zeta=0$, since $U_{0}^{-}=\mathbb{K} 1=U_{0}^{+}$and $(1,1)=1$. Assume now that $\zeta>0$, and suppose that the claim holds for all $\nu$ with $0 \leqslant \nu<\zeta$. Let $x \in U_{\zeta}^{+}$with $(y, x)=0$ for all $y \in U_{-\zeta}^{-}$. In particular, we have, for all $y \in U_{-\left(\zeta-\alpha_{i}\right)}^{-}$, that

$$
\left(f_{i} y, x\right)=0 \quad \text { and } \quad\left(y f_{i}, x\right)=0 \quad \text { for all } 1 \leqslant i<n .
$$

It follows from lemma 4.2(i) and (ii) that $\left(y, p_{i}^{\prime}(x)\right)=0$ and $\left(y, p_{i}(x)\right)=0$. By the induction hypothesis, we have $p_{i}^{\prime}(x)=p_{i}(x)=0$, and it follows from lemma 4.2(iii) that $f_{i} x=x f_{i}$ for all $i$. Lemma 4.4 now applies, to give $x=0$, as desired.

In what follows, $\rho$ will denote the half-sum of the positive roots. Thus,

$$
\rho=\frac{1}{2} \sum_{\alpha>0} \alpha=\sum_{i=1}^{n-1} \varpi_{i}=\frac{1}{2}\left((n-1) \epsilon_{1}+(n-3) \epsilon_{2}+\cdots+((n-1)-2(n-1)) \epsilon_{n}\right) .
$$


It is evident from the triangular decomposition that there is a vector-space isomorphism

$$
\bigoplus_{\mu, \nu \in Q^{+}}\left(U_{-\nu}^{-} \omega_{\nu}^{\prime-1}\right) \otimes U^{0} \otimes U_{\mu}^{+} \stackrel{\sim}{\longrightarrow} U .
$$

This guarantees that the bilinear form which we introduce next is well defined.

Definition 4.6. Set

$$
\left\langle\left(y \omega_{\nu}^{\prime-1}\right) \omega_{\eta}^{\prime} \omega_{\phi} x \mid\left(y_{1} \omega_{\nu_{1}}^{\prime-1}\right) \omega_{\eta_{1}}^{\prime} \omega_{\phi_{1}} x_{1}\right\rangle=\left(y, x_{1}\right)\left(y_{1}, x\right)\left(\omega_{\eta}^{\prime}, \omega_{\phi_{1}}\right)\left(\omega_{\eta_{1}}^{\prime}, \omega_{\phi}\right)\left(r s^{-1}\right)^{\langle\rho, \nu\rangle}
$$

for all $x \in U_{\mu}^{+}, x_{1} \in U_{\mu_{1}}^{+}, y \in U_{-\nu}^{-}, y_{1} \in U_{-\nu_{1}}^{-}, \mu, \mu_{1}, \nu, \nu_{1} \in Q^{+}$, and all $\eta, \eta_{1}, \phi, \phi_{1} \in$ $Q$. Extend this linearly to a bilinear form $\langle\cdot, \cdot\rangle: U \times U \rightarrow \mathbb{K}$ on all of $U$.

Note that

$$
\begin{aligned}
\left\langle\left(y \omega_{\nu}^{\prime-1}\right) \omega_{\eta}^{\prime} \omega_{\phi} x\right| & \left.\left(y_{1} \omega_{\nu_{1}}^{\prime-1}\right) \omega_{\eta_{1}}^{\prime} \omega_{\phi_{1}} x_{1}\right\rangle \\
& =\left\langle y \omega_{\nu}^{\prime-1} \mid x_{1}\right\rangle \cdot\left\langle\omega_{\eta}^{\prime} \omega_{\phi} \mid \omega_{\eta_{1}}^{\prime} \omega_{\phi_{1}}\right\rangle \cdot\left\langle x \mid y_{1} \omega_{\nu_{1}}^{\prime-1}\right\rangle .
\end{aligned}
$$

So the form respects the decomposition

$$
\bigoplus_{\mu, \nu \in Q^{+}}\left(U_{-\nu}^{-} \omega_{\nu}^{\prime-1}\right) \otimes U^{0} \otimes U_{\mu}^{+} \stackrel{\sim}{\rightarrow} U .
$$

The following lemma is an immediate consequence of the above definition and corollary 4.3 .

Lemma 4.7. Assume that $\mu, \mu_{1}, \nu, \nu_{1} \in Q^{+}$. Then

$$
\left\langle U_{-\nu}^{-} U^{0} U_{\mu}^{+} \mid U_{-\nu_{1}}^{-} U^{0} U_{\mu_{1}}^{+}\right\rangle=0
$$

unless $\mu=\nu_{1}$ and $\nu=\mu_{1}$.

Since $U$ is a Hopf algebra, it acts on itself via the adjoint representation,

$$
\operatorname{ad}(u) v=\sum_{(u)} u_{(1)} v S\left(u_{(2)}\right),
$$

where $u, v \in U$ and $\Delta(u)=\sum_{(u)} u_{(1)} \otimes u_{(2)}$.

Proposition 4.8. The bilinear form $\langle\cdot \mid \cdot\rangle$ is ad-invariant, i.e.

$$
\left\langle\operatorname{ad}(u) v \mid v_{1}\right\rangle=\left\langle v \mid \operatorname{ad}(S(u)) v_{1}\right\rangle
$$

for all $u, v, v_{1} \in U$.

Proof. It suffices to assume $u$ is one of the generators $\omega_{i}, \omega_{i}^{\prime}, e_{i}, f_{i}$. Also, without loss of generality, we may suppose that

$$
v=\left(y \omega_{\nu}^{\prime-1}\right) \omega_{\eta}^{\prime} \omega_{\phi} x \quad \text { and } \quad v_{1}=\left(y_{1} \omega_{\nu_{1}}^{\prime-1}\right) \omega_{\eta_{1}}^{\prime} \omega_{\phi_{1}} x_{1}
$$

where $x \in U_{\mu}^{+}, y \in U_{-\nu}^{-}, x_{1} \in U_{\mu_{1}}^{+}, y_{1} \in U_{-\nu_{1}}^{-}$and $\mu, \nu, \mu_{1}, \nu_{1} \in Q^{+}$. 
CASE $1\left(u=\omega_{i}\right)$. From the definition, $\operatorname{ad}\left(\omega_{i}\right) v=\omega_{i} v \omega_{i}^{-1}=r^{\left\langle\epsilon_{i}, \mu-\nu\right\rangle} s^{\left\langle\epsilon_{i+1}, \mu-\nu\right\rangle} v$ so that

$$
\left\langle\operatorname{ad}\left(\omega_{i}\right) v \mid v_{1}\right\rangle=r^{\left\langle\epsilon_{i}, \mu-\nu\right\rangle} s^{\left\langle\epsilon_{i+1}, \mu-\nu\right\rangle}\left\langle v \mid v_{1}\right\rangle
$$

On the other hand, we have

$$
\operatorname{ad}\left(S\left(\omega_{i}\right)\right) v_{1}=\omega_{i}^{-1} v_{1} \omega_{i}=r^{\left\langle\epsilon_{i}, \nu_{1}-\mu_{1}\right\rangle} s^{\left\langle\epsilon_{i+1}, \nu_{1}-\mu_{1}\right\rangle} v_{1},
$$

which implies that

$$
\left\langle v \mid \operatorname{ad}\left(S\left(\omega_{i}\right)\right) v_{1}\right\rangle=r^{\left\langle\epsilon_{i}, \nu_{1}-\mu_{1}\right\rangle} s^{\left\langle\epsilon_{i+1}, \nu_{1}-\mu_{1}\right\rangle}\left\langle v \mid v_{1}\right\rangle
$$

If $\left\langle v \mid v_{1}\right\rangle \neq 0$, then we must have $\nu=\mu_{1}$ and $\nu_{1}=\mu$ by lemma 4.7. Thus, $\mu-\nu=\nu_{1}-\mu_{1}$ and $\left\langle\operatorname{ad}\left(\omega_{i}\right) v \mid v_{1}\right\rangle=\left\langle v \mid \operatorname{ad}\left(S\left(\omega_{i}\right)\right) v_{1}\right\rangle$.

CASE $2\left(u=\omega_{i}^{\prime}\right)$. We have only to replace $\omega_{i}$ by $\omega_{i}^{\prime}$ and interchange $\epsilon_{i}$ and $\epsilon_{i+1}$ in the argument of case 1 .

CASE $3\left(u=e_{i}\right)$. This case is similar to case 4 , below, so we omit the calculation.

CASE $4\left(u=f_{i}\right)$. Using lemmas 2.1 and $4.2($ iii), we get

$$
\begin{aligned}
\operatorname{ad}\left(f_{i}\right) v= & v S\left(f_{i}\right)+f_{i} v S\left(\omega_{i}^{\prime}\right)=-v f_{i}\left(\omega_{i}^{\prime}\right)^{-1}+f_{i} v\left(\omega_{i}^{\prime}\right)^{-1} \\
=- & y\left(\omega_{\nu}^{\prime}\right)^{-1} \omega_{\eta}^{\prime} \omega_{\phi} x f_{i}\left(\omega_{i}^{\prime}\right)^{-1}+f_{i} y\left(\omega_{\nu}^{\prime}\right)^{-1} \omega_{\eta}^{\prime} \omega_{\phi} x\left(\omega_{i}^{\prime}\right)^{-1} \\
=- & y\left(\omega_{\nu}^{\prime}\right)^{-1} \omega_{\eta}^{\prime} \omega_{\phi} f_{i} x\left(\omega_{i}^{\prime}\right)^{-1}+(s-r)^{-1} y\left(\omega_{\nu}^{\prime}\right)^{-1} \omega_{\eta}^{\prime} \omega_{\phi} p_{i}(x) \omega_{i}\left(\omega_{i}^{\prime}\right)^{-1} \\
& \quad-(s-r)^{-1} y\left(\omega_{\nu}^{\prime}\right)^{-1} \omega_{\eta}^{\prime} \omega_{\phi} \omega_{i}^{\prime} p_{i}^{\prime}(x)\left(\omega_{i}^{\prime}\right)^{-1}+f_{i} y\left(\omega_{\nu}^{\prime}\right)^{-1} \omega_{\eta}^{\prime} \omega_{\phi} x\left(\omega_{i}^{\prime}\right)^{-1} \\
=- & r^{\left\langle\epsilon_{i}, \eta-\nu\right\rangle} r^{\left\langle\epsilon_{i+1}, \phi+\mu\right\rangle} s^{\left\langle\epsilon_{i}, \phi+\mu\right\rangle} s^{\left\langle\epsilon_{i+1}, \eta-\nu\right\rangle} y f_{i}\left(\omega_{\nu+\alpha_{i}}^{\prime}\right)^{-1} \omega_{\eta}^{\prime} \omega_{\phi} x \\
& \quad+r^{\left\langle\epsilon_{i+1}, \mu\right\rangle} s^{\left\langle\epsilon_{i}, \mu\right\rangle} f_{i} y\left(\omega_{\nu+\alpha_{i}}^{\prime}\right)^{-1} \omega_{\eta}^{\prime} \omega_{\phi} x \\
& \quad+(s-r)^{-1} r^{-\left\langle\alpha_{i}, \mu-\alpha_{i}\right\rangle} s^{\left\langle\alpha_{i}, \mu-\alpha_{i}\right\rangle} y\left(\omega_{\nu}^{\prime}\right)^{-1} \omega_{\eta-\alpha_{i}}^{\prime} \omega_{\phi+\alpha_{i}} p_{i}(x) \\
& \quad-(s-r)^{-1} r^{\left\langle\epsilon_{i+1}, \mu-\alpha_{i}\right\rangle} s^{\left\langle\epsilon_{i}, \mu-\alpha_{i}\right\rangle} y\left(\omega_{\nu}^{\prime}\right)^{-1} \omega_{\eta}^{\prime} \omega_{\phi} p_{i}^{\prime}(x) .
\end{aligned}
$$

Now

$$
\operatorname{ad}\left(S\left(f_{i}\right)\right) v_{1}=\operatorname{ad}\left(-f_{i}\left(\omega_{i}^{\prime}\right)^{-1}\right) v_{1}=-r^{-\left\langle\epsilon_{i+1}, \mu_{1}-\nu_{1}\right\rangle} s^{-\left\langle\epsilon_{i}, \mu_{1}-\nu_{1}\right\rangle} \operatorname{ad}\left(f_{i}\right) v_{1} .
$$

We apply the previous calculation of $\operatorname{ad}\left(f_{i}\right) v$ with $v$ replaced by $v_{1}$ to see that

$$
\begin{aligned}
\operatorname{ad}\left(S\left(f_{i}\right)\right) v_{1}=r^{\left\langle\epsilon_{i}, \eta_{1}-\nu_{1}\right\rangle} r^{\left\langle\epsilon_{i+1}, \phi_{1}+\nu_{1}\right\rangle} s^{\left\langle\epsilon_{i}, \phi_{1}+\nu_{1}\right\rangle} s^{\left\langle\epsilon_{i+1}, \eta_{1}-\nu_{1}\right\rangle} y_{1} f_{i}\left(\omega_{\nu_{1}+\alpha_{i}}^{\prime}\right)^{-1} \omega_{\eta_{1}}^{\prime} \omega_{\phi_{1}} x_{1} \\
-r^{\left\langle\epsilon_{i+1}, \nu_{1}\right\rangle} s^{\left\langle\epsilon_{i}, \nu_{1}\right\rangle} f_{i} y_{1}\left(\omega_{\nu_{1}+\alpha_{i}}^{\prime}\right)^{-1} \omega_{\eta_{1}}^{\prime} \omega_{\phi_{1}} x_{1} \\
-(s-r)^{-1} r^{-\left\langle\epsilon_{i}, \mu_{1}-\alpha_{i}\right\rangle} r^{\left\langle\epsilon_{i+1}, \nu_{1}-\alpha_{i}\right\rangle} s^{\left\langle\epsilon_{i}, \nu_{1}-\alpha_{i}\right\rangle} s^{-\left\langle\epsilon_{i+1}, \mu_{1}-\alpha_{i}\right\rangle} \\
\quad \times y_{1}\left(\omega_{\nu_{1}}^{\prime}\right)^{-1} \omega_{\eta_{1}-\alpha_{i}}^{\prime} \omega_{\phi_{1}+\alpha_{i}} p_{i}\left(x_{1}\right) \\
+(s-r)^{-1} r^{\left\langle\epsilon_{i+1}, \nu_{1}-\alpha_{i}\right\rangle} s^{\left\langle\epsilon_{i}, \nu_{1}-\alpha_{i}\right\rangle} y_{1}\left(\omega_{\nu_{1}}^{\prime}\right)^{-1} \omega_{\eta_{1}}^{\prime} \omega_{\phi_{1}} p_{i}^{\prime}\left(x_{1}\right) .
\end{aligned}
$$

It follows from lemma 4.7 that $\left\langle\operatorname{ad}\left(f_{i}\right) v \mid v_{1}\right\rangle$ and $\left\langle v \mid \operatorname{ad}\left(S\left(f_{i}\right)\right) v_{1}\right\rangle$ can be non-zero when either (a) $\nu+\alpha_{i}=\mu_{1}$ and $\nu_{1}=\mu$, or (b) $\nu=\mu_{1}$ and $\nu_{1}=\mu-\alpha_{i}$. 
(a) By lemma 4.2(i), (ii), we have

$$
\begin{gathered}
\left\langle\operatorname{ad}\left(f_{i}\right) v \mid v_{1}\right\rangle=-r^{\left\langle\epsilon_{i}, \eta-\nu\right\rangle} r^{\left\langle\epsilon_{i+1}, \phi+\mu\right\rangle} s^{\left\langle\epsilon_{i}, \phi+\mu\right\rangle} s^{\left\langle\epsilon_{i+1}, \eta-\nu\right\rangle} \\
\quad \times\left(y f_{i}, x_{1}\right)\left(y_{1}, x\right)\left(\omega_{\eta}^{\prime}, \omega_{\phi_{1}}\right)\left(\omega_{\eta_{1}}^{\prime}, \omega_{\phi}\right)\left(r s^{-1}\right)^{\left\langle\rho, \nu+\alpha_{i}\right\rangle} \\
+r^{\left\langle\epsilon_{i+1}, \mu\right\rangle} s^{\left\langle\epsilon_{i}, \mu\right\rangle}\left(f_{i} y, x_{1}\right)\left(y_{1}, x\right)\left(\omega_{\eta}^{\prime}, \omega_{\phi_{1}}\right)\left(\omega_{\eta_{1}}^{\prime}, \omega_{\phi}\right)\left(r s^{-1}\right)^{\left\langle\rho, \nu+\alpha_{i}\right\rangle} \\
=A \times\left(y_{1}, x\right)\left(\omega_{\eta}^{\prime}, \omega_{\phi_{1}}\right)\left(\omega_{\eta_{1}}^{\prime}, \omega_{\phi}\right)\left(r s^{-1}\right)^{\langle\rho, \nu\rangle},
\end{gathered}
$$

where

$$
\begin{aligned}
A=-(s-r)^{-1} r^{\left\langle\epsilon_{i}, \eta-\nu\right\rangle} r^{\left\langle\epsilon_{i+1}, \phi+\mu\right\rangle} s^{\left\langle\epsilon_{i}, \phi+\mu\right\rangle} s^{\left\langle\epsilon_{i+1}, \eta-\nu\right\rangle} & r s^{-1}\left(y, p_{i}\left(x_{1}\right)\right) \\
& +(s-r)^{-1} r^{\left\langle\epsilon_{i+1}, \mu\right\rangle} s^{\left\langle\epsilon_{i}, \mu\right\rangle} r s^{-1}\left(y, p_{i}^{\prime}\left(x_{1}\right)\right) .
\end{aligned}
$$

Similarly,

$$
\left\langle v \mid \operatorname{ad}\left(S\left(f_{i}\right)\right) v_{1}\right\rangle=B \times\left(y_{1}, x\right)\left(\omega_{\eta}^{\prime}, \omega_{\phi_{1}}\right)\left(\omega_{\eta_{1}}^{\prime}, \omega_{\phi}\right)\left(r s^{-1}\right)^{\langle\rho, \nu\rangle},
$$

where

$$
\begin{aligned}
B=-(s-r)^{-1} r^{-\left\langle\epsilon_{i}, \mu_{1}-\alpha_{i}\right\rangle} r^{\left\langle\epsilon_{i+1}, \nu_{1}-\alpha_{i}\right\rangle} s^{\left\langle\epsilon_{i}, \nu_{1}-\alpha_{i}\right\rangle} s^{-\left\langle\epsilon_{i+1}, \mu_{1}-\alpha_{i}\right\rangle} & \\
& \times\left(\omega_{\eta}^{\prime}, \omega_{i}\right)\left(\left(\omega_{i}^{\prime}\right)^{-1}, \omega_{\phi}\right)\left(y, p_{i}\left(x_{1}\right)\right) \\
& +(s-r)^{-1} r^{\left\langle\epsilon_{i+1}, \nu_{1}-\alpha_{i}\right\rangle} s^{\left\langle\epsilon_{i}, \nu_{1}-\alpha_{i}\right\rangle}\left(y, p_{i}^{\prime}\left(x_{1}\right)\right) .
\end{aligned}
$$

Comparing both sides, we conclude that $\left\langle\operatorname{ad}\left(f_{i}\right) v \mid v_{1}\right\rangle=\left\langle v \mid \operatorname{ad}\left(S\left(f_{i}\right)\right) v_{1}\right\rangle$.

(b) An argument analogous to that for (a) can be used in this case.

REMARK 4.9. It was shown in [4] that $U$ is isomorphic to the Drinfel'd double $D\left(B,\left(B^{\prime}\right)^{\text {coop }}\right)$, where $B$ is the Hopf subalgebra of $U$ generated by the elements $\omega_{j}^{ \pm 1}, e_{j}, 1 \leqslant j<n$, and $\left(B^{\prime}\right)^{\text {coop }}$ is the subalgebra of $U$ generated by the elements $\left(\omega_{j}^{\prime}\right)^{ \pm 1}, f_{j}, 1 \leqslant j<n$, but with the opposite co-product. This realization of $U$ allows us to define the Rosso form $R$ on $U$ according to [18, p. 77]:

$$
R\left\langle a \otimes b \mid a^{\prime} \otimes b^{\prime}\right\rangle=\left(b^{\prime}, S(a)\right)\left(S^{-1}(b), a^{\prime}\right) \quad \text { for } a, a^{\prime} \in B \text { and } b, b^{\prime} \in\left(B^{\prime}\right)^{\text {coop }} .
$$

The Rosso form is also an ad-invariant form on $U$, but it does not admit the decomposition in (4.3). Rather, it has the following factorization (we suppress the tensor symbols in the notation):

$$
\begin{aligned}
& R\left\langle x \omega_{\phi} \omega_{\eta}^{\prime}\left(\omega_{\nu}^{\prime-1} y\right) \mid x_{1} \omega_{\phi_{1}} \omega_{\eta_{1}}^{\prime}\left(\omega_{\nu_{1}}^{\prime}{ }^{-1} y_{1}\right)\right\rangle \\
& =R\left\langle x \mid \omega_{\nu_{1}}^{\prime}{ }^{-1} y_{1}\right\rangle \cdot R\left\langle\omega_{\phi} \omega_{\eta}^{\prime} \mid \omega_{\phi_{1}} \omega_{\eta_{1}}^{\prime}\right\rangle \cdot R\left\langle\omega_{\nu}^{\prime-1} y \mid x_{1}\right\rangle .
\end{aligned}
$$

That is to say, the form $R$ respects the decomposition

$$
\bigoplus_{\mu, \nu \in Q^{+}} U_{\mu}^{+} \otimes U^{0} \otimes\left(\omega_{\nu}^{\prime-1} U_{-\nu}^{-}\right) \stackrel{\sim}{\rightarrow} U .
$$

For $(\eta, \phi) \in Q \times Q$, we define a group homomorphism $\chi_{\eta, \phi}: Q \times Q \rightarrow \mathbb{K}^{\times}$by

$$
\chi_{\eta, \phi}\left(\eta_{1}, \phi_{1}\right)=\left(\omega_{\eta}^{\prime}, \omega_{\phi_{1}}\right)\left(\omega_{\eta_{1}}^{\prime}, \omega_{\phi}\right), \quad\left(\eta_{1}, \phi_{1}\right) \in Q \times Q .
$$


LEMMA 4.10. Assume that $r^{k} s^{l}=1$ if and only if $k=l=0$. If $\chi_{\eta, \phi}=\chi_{\eta^{\prime}, \phi^{\prime}}$, then $(\eta, \phi)=\left(\eta^{\prime}, \phi^{\prime}\right)$.

Proof. If $\chi_{\eta, \phi}=\chi_{\eta^{\prime}, \phi^{\prime}}$, then

$$
\chi_{\eta, \phi}\left(0, \alpha_{j}\right)=r^{\left\langle\epsilon_{j}, \eta\right\rangle} s^{\left\langle\epsilon_{j+1}, \eta\right\rangle}=\chi_{\eta^{\prime}, \phi^{\prime}}\left(0, \alpha_{j}\right)=r^{\left\langle\epsilon_{j}, \eta^{\prime}\right\rangle} s^{\left\langle\epsilon_{j+1}, \eta^{\prime}\right\rangle} .
$$

Since $r^{\left\langle\epsilon_{j}, \eta\right\rangle-\left\langle\epsilon_{j}, \eta^{\prime}\right\rangle} s^{\left\langle\epsilon_{j+1}, \eta\right\rangle-\left\langle\epsilon_{j+1}, \eta^{\prime}\right\rangle}=1$, it must be that $\left\langle\epsilon_{j}, \eta\right\rangle=\left\langle\epsilon_{j}, \eta^{\prime}\right\rangle$ for all $1 \leqslant j \leqslant n$. From this it is easy to see that $\eta=\eta^{\prime}$. Similar considerations with $\chi_{\eta, \phi}\left(\alpha_{i}, 0\right)=\chi_{\eta^{\prime}, \phi^{\prime}}\left(\alpha_{i}, 0\right)$ show that $\phi=\phi^{\prime}$.

Proposition 4.11. Assume that $r^{k} s^{l}=1$ if and only if $k=l=0$. Then the bilinear form $\langle\cdot \mid \cdot\rangle$ is non-degenerate on $U$.

Proof. It is sufficient to argue that if $u \in U_{-\nu}^{-} U^{0} U_{\mu}^{+}$and $\langle u \mid v\rangle=0$ for all $v \in$ $U_{-\mu}^{-} U^{0} U_{\nu}^{+}$, then $u=0$. Choose, for each $\mu \in Q^{+}$, a basis $u_{1}^{\mu}, u_{2}^{\mu}, \ldots, u_{d_{\mu}}^{\mu}, d_{\mu}=$ $\operatorname{dim} U_{\mu}^{+}$, of $U_{\mu}^{+}$. Owing to lemma 4.5 , we can take a dual basis $v_{1}^{\mu}, v_{2}^{\mu}, \ldots, v_{d_{\mu}}^{\mu}$ of $U_{-\mu}^{-}$, i.e. $\left(v_{i}^{\mu}, u_{j}^{\mu}\right)=\delta_{i, j}$. Then the set

$$
\left\{\left(v_{i}^{\nu} \omega_{\nu}^{\prime-1}\right) \omega_{\eta}^{\prime} \omega_{\phi} u_{j}^{\mu} \mid 1 \leqslant i \leqslant d_{\nu}, 1 \leqslant j \leqslant d_{\mu} \text { and } \eta, \phi \in Q\right\}
$$

is a basis of $U_{-\nu}^{-} U^{0} U_{\mu}^{+}$. From the definition of the bilinear form, we obtain

$$
\begin{aligned}
\left\langle\left(v_{i}^{\nu} \omega_{\nu}^{\prime-1}\right) \omega_{\eta}^{\prime} \omega_{\phi} u_{j}^{\mu}\right| & \left.\left(v_{k}^{\mu} \omega_{\mu}^{\prime-1}\right) \omega_{\eta_{1}} \omega_{\phi_{1}} u_{l}^{\nu}\right\rangle \\
& =\left(v_{i}^{\nu}, u_{l}^{\nu}\right)\left(v_{k}^{\mu}, u_{j}^{\mu}\right)\left(\omega_{\eta}^{\prime}, \omega_{\phi_{1}}\right)\left(\omega_{\eta_{1}}^{\prime}, \omega_{\phi}\right)\left(r s^{-1}\right)^{\langle\rho, \nu\rangle} \\
& =\delta_{i, l} \delta_{j, k}\left(\omega_{\eta}^{\prime}, \omega_{\phi_{1}}\right)\left(\omega_{\eta_{1}}^{\prime}, \omega_{\phi}\right)\left(r s^{-1}\right)^{\langle\rho, \nu\rangle} .
\end{aligned}
$$

Now write $u=\sum_{i, j, \eta, \phi} \theta_{i, j, \eta, \phi}\left(v_{i}^{\nu} \omega_{\nu}^{\prime-1}\right) \omega_{\eta}^{\prime} \omega_{\phi} u_{j}^{\mu}$, and take $v=\left(v_{k}^{\mu} \omega_{\mu}^{\prime-1}\right) \omega_{\eta_{1}}^{\prime} \omega_{\phi_{1}} u_{l}^{\nu}$ with $1 \leqslant k \leqslant d_{\mu}$ and $1 \leqslant l \leqslant d_{\nu}$ and $\eta_{1}, \phi_{1} \in Q$. From the assumption $\langle u \mid v\rangle=0$ we have

$$
\sum_{\eta, \phi} \theta_{l, k, \eta, \phi}\left(\omega_{\eta}^{\prime}, \omega_{\phi_{1}}\right)\left(\omega_{\eta_{1}}^{\prime}, \omega_{\phi}\right)\left(r s^{-1}\right)^{\langle\rho, \nu\rangle}=0
$$

for all $1 \leqslant k \leqslant d_{\mu}$ and $1 \leqslant l \leqslant d_{\nu}$ and for all $\eta_{1}, \phi_{1} \in Q$. Equation (4.6) can be written as

$$
\sum_{\eta, \phi} \theta_{l, k, \eta, \phi}\left(r s^{-1}\right)^{\langle\rho, \nu\rangle} \chi_{\eta, \phi}=0
$$

for each $k$ and $l$ (where $1 \leqslant k \leqslant d_{\mu}$ and $1 \leqslant l \leqslant d_{\nu}$ ). It follows from lemma 4.10 and the linear independence of distinct characters (Dedekind's theorem; see, for example, [17, p. 280]) that $\theta_{l, k, \eta, \phi}=0$ for all $\eta, \phi \in Q$ and for all $l$ and $k$. Hence, we have $u=0$ as desired.

\section{The centre of $U=U_{r, s}\left(\mathfrak{s l}_{n}\right)$}

Throughout this section we make the following assumption:

$$
r^{k} s^{l}=1 \quad \text { if and only if } k=l=0 .
$$

Under this hypothesis, we see that, for $\zeta \in Q$,

$$
U_{\zeta}=\left\{z \in U \mid \omega_{i} z \omega_{i}^{-1}=r^{\left\langle\epsilon_{i}, \zeta\right\rangle} s^{\left\langle\epsilon_{i+1}, \zeta\right\rangle} z \text { and } \omega_{i}^{\prime} z\left(\omega_{i}^{\prime}\right)^{-1}=r^{\left\langle\epsilon_{i+1}, \zeta\right\rangle} s^{\left\langle\epsilon_{i}, \zeta\right\rangle} z\right\} .
$$


We denote the centre of $U$ by $\mathfrak{Z}$. Since any central element of $U$ must commute with $\omega_{i}$ and $\omega_{i}^{\prime}$ for all $i$, it follows from (5.2) that $\mathfrak{Z} \subset U_{0}$. We define an algebra automorphism $\gamma^{-\rho}: U^{0} \rightarrow U^{0}$ by

$$
\gamma^{-\rho}\left(a_{i}\right)=r^{-\left\langle\rho, \epsilon_{i}\right\rangle} a_{i} \quad \text { and } \quad \gamma^{-\rho}\left(b_{i}\right)=s^{-\left\langle\rho, \epsilon_{i}\right\rangle} b_{i} .
$$

Thus,

$$
\gamma^{-\rho}\left(\omega_{i}^{\prime} \omega_{i}^{-1}\right)=\left(r s^{-1}\right)^{\left\langle\rho, \alpha_{i}\right\rangle} \omega_{i}^{\prime} \omega_{i}^{-1} .
$$

Definition 5.1. The Harish-Chandra homomorphism $\xi: \mathfrak{Z} \rightarrow U^{0}$ is the restriction to $\mathfrak{Z}$ of the map

$$
\gamma^{-\rho} \circ \pi: U_{0} \stackrel{\pi}{\rightarrow} U^{0} \stackrel{\gamma^{-\rho}}{\longrightarrow} U^{0},
$$

where $\pi: U_{0} \rightarrow U^{0}$ is the canonical projection.

Proposition 5.2. $\xi$ is an injective algebra homomorphism.

Proof. Note that $U_{0}=U^{0} \oplus K$, where $K=\bigoplus_{\nu>0} U_{-\nu}^{-} U^{0} U_{\nu}^{+}$is the two-sided ideal in $U_{0}$ which is the kernel of $\pi$, and hence of $\xi$. Thus, $\xi$ is an algebra homomorphism. Assume that $z \in \mathfrak{Z}$ and $\xi(z)=0$. Writing $z=\sum_{\nu \in Q^{+}} z_{\nu}$ with $z_{\nu} \in U_{-\nu}^{-} U^{0} U_{\nu}^{+}$, we have $z_{0}=0$. Fix any $\nu \in Q^{+} \backslash\{0\}$ minimal with the property that $z_{\nu} \neq 0$. Also choose bases $\left\{y_{k}\right\}$ and $\left\{x_{l}\right\}$ for $U_{-\nu}^{-}$and $U_{\nu}^{+}$, respectively. We may write $z_{\nu}=\sum_{k, l} y_{k} t_{k, l} x_{l}$ for some $t_{k, l} \in U^{0}$. Then

$$
\begin{aligned}
0 & =e_{i} z-z e_{i} \\
& =\sum_{\gamma \neq \nu}\left(e_{i} z_{\gamma}-z_{\gamma} e_{i}\right)+\sum_{k, l}\left(e_{i} y_{k}-y_{k} e_{i}\right) t_{k, l} x_{l}+\sum_{k, l} y_{k}\left(e_{i} t_{k, l} x_{l}-t_{k, l} x_{l} e_{i}\right) .
\end{aligned}
$$

Note that $e_{i} y_{k}-y_{k} e_{i} \in U_{-\left(\nu-\alpha_{i}\right)}^{-} U^{0}$. Recalling the minimality of $\nu$, we see that only the second term belongs to $U_{-\left(\nu-\alpha_{i}\right)}^{-\alpha_{i}} U^{0} U_{\nu}^{+}$. Therefore, we have

$$
\sum_{k, l}\left(e_{i} y_{k}-y_{k} e_{i}\right) t_{k, l} x_{l}=0 .
$$

By the triangular decomposition of $U$ and the fact that $\left\{x_{l}\right\}$ is a basis of $U_{\nu}^{+}$, we get $\sum_{k} e_{i} y_{k} t_{k, l}=\sum_{k} y_{k} e_{i} t_{k, l}$ for each $l$ and for all $1 \leqslant i<n$.

Now we fix $l$ and consider the irreducible module $L(\lambda)$ for $\lambda \in \Lambda_{\mathfrak{s l}}^{+}$. Let $v_{\lambda}$ be the highest weight vector of $L(\lambda)$, and set $m=\sum_{k} y_{k} t_{k, l} v_{\lambda}$. Then, for each $i$,

$$
e_{i} m=\sum_{k} e_{i} y_{k} t_{k, l} v_{\lambda}=\sum_{k} y_{k} e_{i} t_{k, l} v_{\lambda}=0 .
$$

Hence, $m$ generates a proper submodule of $L(\lambda)$. The irreducibility of $L(\lambda)$ forces $m=0$. Choosing an appropriate $\lambda \in \Lambda_{\mathfrak{s l}}^{+}$with lemma 2.2 in mind, we have

$$
\sum_{k} y_{k} t_{k, l}=0
$$

Since $\left\{y_{k}\right\}$ is a basis for $U_{-\nu}^{-}$, it must be that $t_{k, l}=0$ for each $k$. But $l$ can be arbitrary, so we get $z_{\nu}=0$, which is a contradiction. 
Proposition 5.3. If $n$ is even, set

$$
\mathfrak{z}=\omega_{1}^{\prime} \omega_{3}^{\prime} \cdots \omega_{n-1}^{\prime} \omega_{1} \omega_{3} \cdots \omega_{n-1}=a_{1} \cdots a_{n} b_{1} \cdots b_{n} .
$$

Then $\mathfrak{z}$ is central and $\xi(\mathfrak{z})=\mathfrak{z}$.

Proof. We have

$$
e_{i \mathfrak{z}}=r^{-\left\langle\epsilon_{1}+\epsilon_{2}+\cdots+\epsilon_{n}, \alpha_{i}\right\rangle} s^{-\left\langle\epsilon_{1}+\epsilon_{2}+\cdots+\epsilon_{n}, \alpha_{i}\right\rangle} \mathfrak{z} e_{i}=\mathfrak{z} e_{i} \quad \text { for all } 1 \leqslant i<n .
$$

Similarly, $f_{i \mathfrak{z}}=\mathfrak{z} f_{i}$ for all $1 \leqslant i<n$, so that $\mathfrak{z}$ is central. Finally, observe that

$$
\xi(\mathfrak{z})=r^{-\left\langle\rho, \epsilon_{1}+\epsilon_{2}+\cdots+\epsilon_{n}\right\rangle} s^{-\left\langle\rho, \epsilon_{1}+\epsilon_{2}+\cdots+\epsilon_{n}\right\rangle} \mathfrak{z}=\mathfrak{z} .
$$

By introducing appropriate factors into the definition of the homomorphism $\varrho^{\lambda}$ in (2.2), we are able to obtain a duality between $U^{0}$ and its characters. Thus, for any $\lambda, \mu \in \Lambda_{\mathfrak{s l}}$, we let $\varrho^{\lambda, \mu}: U^{0} \rightarrow \mathbb{K}$ be the algebra homomorphism defined by

$$
\left.\begin{array}{l}
\varrho^{\lambda, \mu}\left(\omega_{j}\right)=r^{\left\langle\epsilon_{j}, \lambda\right\rangle} s^{\left\langle\epsilon_{j+1}, \lambda\right\rangle}\left(r s^{-1}\right)^{\left\langle\alpha_{j}, \mu\right\rangle}, \\
\varrho^{\lambda, \mu}\left(\omega_{j}^{\prime}\right)=r^{\left\langle\epsilon_{j+1}, \lambda\right\rangle} s^{\left\langle\epsilon_{j}, \lambda\right\rangle}\left(r s^{-1}\right)^{\left\langle\alpha_{j}, \mu\right\rangle} .
\end{array}\right\}
$$

In particular, $\varrho^{\lambda, 0}$ is just the homomorphism $\varrho^{\lambda}$ on $U^{0}$.

Lemma 5.4. Assume that $u=\omega_{\eta}^{\prime} \omega_{\phi}$ with $\eta, \phi \in Q$. If $\varrho^{\lambda, \mu}(u)=1$ for all $\lambda, \mu \in \Lambda_{\mathfrak{s l}}$, then $u=1$.

Proof. We write $\eta=\sum_{i} \eta_{i} \alpha_{i}$ and $\phi=\sum_{i} \phi_{i} \alpha_{i}$. Then $\varrho^{\varpi_{i}, 0}(u)=\varrho^{\varpi_{i}, 0}\left(\omega_{\eta}^{\prime} \omega_{\phi}\right)=$ $r^{A_{i}} s^{B_{i}}=1$ for each $1 \leqslant i<n$, where

$$
\begin{aligned}
& A_{i}=\left\langle\epsilon_{2}, \varpi_{i}\right\rangle \eta_{1}+\cdots+\left\langle\epsilon_{n}, \varpi_{i}\right\rangle \eta_{n-1}+\left\langle\epsilon_{1}, \varpi_{i}\right\rangle \phi_{1}+\cdots+\left\langle\epsilon_{n-1}, \varpi_{i}\right\rangle \phi_{n-1}, \\
& B_{i}=\left\langle\epsilon_{1}, \varpi_{i}\right\rangle \eta_{1}+\cdots+\left\langle\epsilon_{n-1}, \varpi_{i}\right\rangle \eta_{n-1}+\left\langle\epsilon_{2}, \varpi_{i}\right\rangle \phi_{1}+\cdots+\left\langle\epsilon_{n}, \varpi_{i}\right\rangle \phi_{n-1} .
\end{aligned}
$$

It follows from assumption (5.1) that $A_{i}=B_{i}=0$. It is now straightforward to see from the definitions that, for $1 \leqslant i<n$,

$$
\begin{aligned}
& A_{i}=\sum_{j=1}^{i-1} \eta_{j}-\frac{i}{n} \sum_{j=1}^{n-1} \eta_{j}+\sum_{j=1}^{i} \phi_{j}-\frac{i}{n} \sum_{j=1}^{n-1} \phi_{j}=0, \\
& B_{i}=\sum_{j=1}^{i} \eta_{j}-\frac{i}{n} \sum_{j=1}^{n-1} \eta_{j}+\sum_{j=1}^{i-1} \phi_{j}-\frac{i}{n} \sum_{j=1}^{n-1} \phi_{j}=0 .
\end{aligned}
$$

After elementary manipulations we have $\eta_{i}=\phi_{i}$ for all $1 \leqslant i<n$ and $\eta_{2}=\eta_{4}=$ $\cdots=0$ and

$$
\eta_{1}=\eta_{3}=\cdots=\frac{2}{n} \sum_{j=1}^{n-1} \eta_{j}=\frac{2}{n} l \eta_{1},
$$

where $l=\frac{1}{2} n$ if $n$ is even and $l=\frac{1}{2}(n-1)$ if $n$ is odd. Therefore, $u=1$ when $n$ is odd, and $u=\mathfrak{z}^{\eta_{1}}, \eta_{1} \in \mathbb{Z}$, when $n$ is even. Now, when $n$ is even,

$$
1=\varrho^{0, \varpi_{1}}(u)=\left(\varrho^{0, \varpi_{1}}(\mathfrak{z})\right)^{\eta_{1}}=\left(r s^{-1}\right)^{2 \eta_{1}} .
$$

Thus, $\eta_{1}=0$, and $u=1$ as desired. 
Corollary 5.5. Assume that $u \in U^{0}$. If $\varrho^{\lambda, \mu}(u)=0$ for all $(\lambda, \mu) \in \Lambda_{\mathfrak{s l}} \times \Lambda_{\mathfrak{s l}}$, then $u=0$.

Proof. Corresponding to each $(\eta, \phi) \in Q \times Q$ is the character on the group $\Lambda_{\mathfrak{s l}} \times \Lambda_{\mathfrak{s l}}$ defined by

$$
(\lambda, \mu) \mapsto \varrho^{\lambda, \mu}\left(\omega_{\eta}^{\prime} \omega_{\phi}\right) .
$$

It follows from lemma 5.4 that different $(\eta, \phi)$ give rise to different characters.

Suppose now that $u=\sum \theta_{\eta, \phi} \omega_{\eta}^{\prime} \omega_{\phi}$, where $\theta_{\eta, \phi} \in \mathbb{K}$. By assumption,

$$
\sum \theta_{\eta, \phi} \varrho^{\lambda, \mu}\left(\omega_{\eta}^{\prime} \omega_{\phi}\right)=0
$$

for all $(\lambda, \mu) \in \Lambda_{\mathfrak{s l}} \times \Lambda_{\mathfrak{s l}}$. By the linear independence of different characters, $\theta_{\eta, \phi}=0$ for all $(\eta, \phi) \in Q \times Q$, and so $u=0$.

Set

$$
\begin{aligned}
& U_{b}^{0}=\bigoplus_{\eta \in Q} \mathbb{K} \omega_{\eta}^{\prime} \omega_{-\eta}, \\
& U_{\natural}^{0}= \begin{cases}U_{b}^{0} & \text { if } n \text { is odd, } \\
\bigoplus \mathbb{K} \omega_{\eta}^{\prime} \omega_{\phi}, & \text { if } n \text { is even, }\end{cases}
\end{aligned}
$$

where, in the even case, the sum is over the pairs $(\eta, \phi) \in Q \times Q$ which satisfy the following condition: if $\eta=\sum_{i=1}^{n-1} \eta_{i} \alpha_{i}$ and $\phi=\sum_{i=1}^{n-1} \phi_{i} \alpha_{i}$, then

$$
\left.\begin{array}{l}
\eta_{1}+\phi_{1}=\eta_{3}+\phi_{3}=\cdots=\eta_{n-1}+\phi_{n-1}, \\
\eta_{2}+\phi_{2}=\eta_{4}+\phi_{4}=\cdots=\eta_{n-2}+\phi_{n-2}=0 .
\end{array}\right\}
$$

Clearly, $U_{b}^{0} \subsetneq U_{\natural}^{0}$ when $n$ is even, as $\mathfrak{z} \in U_{\natural}^{0} \backslash U_{b}^{0}$.

There is an action of the Weyl group $W$ on $U^{0}$ defined by

$$
\sigma\left(a_{\lambda} b_{\mu}\right)=a_{\sigma(\lambda)} b_{\sigma(\mu)}
$$

for all $\lambda, \mu \in \Lambda$ and $\sigma \in W$. We want to know the effect of this action on a product $\omega_{\eta}^{\prime} \omega_{\phi}$, where $\eta=\sum_{i=1}^{n-1} \eta_{i} \alpha_{i}$ and $\phi=\sum_{i=1}^{n-1} \phi_{i} \alpha_{i}$. For this, write $\omega_{\eta}^{\prime} \omega_{\phi}=a_{\mu} b_{\nu}$, where $\mu=\sum_{i=1}^{n} \mu_{i} \epsilon_{i}, \nu=\sum_{i=1}^{n} \nu_{i} \epsilon_{i}$, and

$$
\mu_{i}=\eta_{i-1}+\phi_{i}, \quad \nu_{i}=\eta_{i}+\phi_{i-1}
$$

for all $1 \leqslant i \leqslant n$ (where $\eta_{0}=\eta_{n}=\phi_{0}=\phi_{n}=0$ ). Then, for the simple reflection $\sigma_{k}$, we have

$$
\begin{aligned}
\sigma_{k}\left(\omega_{\eta}^{\prime} \omega_{\phi}\right) & =\sigma_{k}\left(a_{\mu} b_{\nu}\right) \\
& =a_{\mu} b_{\nu} a_{\alpha_{k}}^{-\left\langle\mu, \alpha_{k}\right\rangle} b_{\alpha_{k}}^{-\left\langle\nu, \alpha_{k}\right\rangle} \\
& =\omega_{\eta}^{\prime} \omega_{\phi}\left(a_{k} a_{k+1}^{-1}\right)^{-\left\langle\mu, \alpha_{k}\right\rangle}\left(b_{k} b_{k+1}^{-1}\right)^{-\left\langle\nu, \alpha_{k}\right\rangle} \\
& =\omega_{\eta}^{\prime} \omega_{\phi}\left(a_{k} b_{k+1}\right)^{-\left\langle\mu, \alpha_{k}\right\rangle}\left(a_{k+1} b_{k}\right)^{\left\langle\mu, \alpha_{k}\right\rangle}\left(b_{k}^{-1} b_{k+1}\right)^{\left\langle\mu+\nu, \alpha_{k}\right\rangle} \\
& =\omega_{\eta}^{\prime} \omega_{\phi}\left(\omega_{k}^{\prime} \omega_{k}^{-1}\right)^{\mu_{k}-\mu_{k+1}}\left(b_{k}^{-1} b_{k+1}\right)^{\mu_{k}+\nu_{k}-\mu_{k+1}-\nu_{k+1}} \\
& =\omega_{\eta}^{\prime} \omega_{\phi}\left(\omega_{k}^{\prime} \omega_{k}^{-1}\right)^{\eta_{k-1}-\eta_{k}+\phi_{k}-\phi_{k+1}}\left(b_{k}^{-1} b_{k+1}\right)^{\eta_{k-1}+\phi_{k-1}-\eta_{k+1}-\phi_{k+1}} .
\end{aligned}
$$


From this it is apparent that the subalgebras $U_{\mathrm{b}}^{0}$ and $U_{\mathrm{b}}^{0}$ of $U^{0}$ are closed under the $W$-action. Moreover, the $W$-action on $U_{b}^{0}$ amounts to

$$
\sigma\left(\omega_{\eta}^{\prime} \omega_{-\eta}\right)=\omega_{\sigma(\eta)}^{\prime} \omega_{-\sigma(\eta)} \quad \text { for all } \sigma \in W \text { and } \eta \in Q .
$$

Proposition 5.6. We have

$$
\varrho^{\sigma(\lambda), \mu}(u)=\varrho^{\lambda, \mu}\left(\sigma^{-1}(u)\right)
$$

for all $u \in U_{\natural}^{0}, \sigma \in W$ and $\lambda, \mu \in \Lambda_{\mathfrak{s l}}$.

Proof. First, we show that $\varrho^{\sigma(\lambda), 0}(u)=\varrho^{\lambda, 0}\left(\sigma^{-1}(u)\right)$. Since

$$
\varrho^{\sigma_{i}\left(\varpi_{j}\right), 0}\left(a_{k}\right)=r^{\left\langle\epsilon_{k}, \sigma_{i}\left(\varpi_{j}\right)\right\rangle}=r^{\left\langle\sigma_{i}\left(\epsilon_{k}\right), \varpi_{j}\right\rangle}=\varrho^{\varpi_{j}, 0}\left(\sigma_{i}\left(a_{k}\right)\right)
$$

and

$$
\varrho^{\sigma_{i}\left(\varpi_{j}\right), 0}\left(b_{k}\right)=s^{\left\langle\epsilon_{k}, \sigma_{i}\left(\varpi_{j}\right)\right\rangle}=s^{\left\langle\sigma_{i}\left(\epsilon_{k}\right), \varpi_{j}\right\rangle}=\varrho^{\varpi_{j}, 0}\left(\sigma_{i}\left(b_{k}\right)\right)
$$

for $1 \leqslant i, j<n$ and $1 \leqslant k \leqslant n$, we see that (5.13) holds in this case. Next we argue that $\varrho^{0, \mu}(u)=\varrho^{0, \mu}\left(\sigma^{-1}(u)\right)$. It is sufficient to suppose that $u=\omega_{\eta}^{\prime} \omega_{\phi}$ and $\sigma=\sigma_{k}$ for some $k$. Then (5.12) shows that

$$
\sigma_{k}\left(\omega_{\eta}^{\prime} \omega_{\phi}\right)=\omega_{\eta}^{\prime} \omega_{\phi}\left(\omega_{k}^{\prime} \omega_{k}^{-1}\right)^{\eta_{k-1}-\eta_{k}+\phi_{k}-\phi_{k+1}} .
$$

Now, using the definition of $\varrho^{0, \mu}$, we have $\varrho^{0, \mu}\left(\sigma_{k}\left(\omega_{\eta}^{\prime} \omega_{\phi}\right)\right)=\varrho^{0, \mu}\left(\omega_{\eta}^{\prime} \omega_{\phi}\right)$. Finally, since $\varrho^{\lambda, \mu}(u)=\varrho^{\lambda, 0}(u) \varrho^{0, \mu}(u)$, the assertion follows.

We define

$$
\left(U_{\natural}^{0}\right)^{W}=\left\{u \in U_{\natural}^{0} \mid \sigma(u)=u, \forall \sigma \in W\right\} \quad \text { and } \quad\left(U_{b}^{0}\right)^{W}=U_{\mathrm{b}}^{0} \cap\left(U_{\natural}^{0}\right)^{W} .
$$

LemMA 5.7. Assume that $u \in U^{0}$ and $\varrho^{\lambda, \mu}(u)=\varrho^{\sigma(\lambda), \mu}(u)$ for all $\lambda, \mu \in \Lambda_{\mathfrak{s l}}$ and $\sigma \in W$. Then $u \in\left(U_{\natural}^{0}\right)^{W}$.

Proof. Suppose that $u=\sum_{(\eta, \phi)} \theta_{\eta, \phi} \omega_{\eta}^{\prime} \omega_{\phi} \in U^{0}$ satisfies $\varrho^{\lambda, \mu}(u)=\varrho^{\sigma(\lambda), \mu}(u)$ for all $\lambda, \mu \in \Lambda_{\mathfrak{s l}}$ and $\sigma \in W$. Then

$$
\sum_{(\eta, \phi)} \theta_{\eta, \phi} \varrho^{\lambda, \mu}\left(\omega_{\eta}^{\prime} \omega_{\phi}\right)=\sum_{(\zeta, \psi)} \theta_{\zeta, \psi} \varrho^{\sigma_{i}(\lambda), \mu}\left(\omega_{\zeta}^{\prime} \omega_{\psi}\right)
$$

for all $\lambda, \mu \in \Lambda_{\mathfrak{s l}}$. If $\kappa_{\eta, \phi}$ and $\kappa_{\zeta, \psi}^{i}$ are the characters on $\Lambda_{\mathfrak{s l}} \times \Lambda_{\mathfrak{s l}}$ defined by

$$
\kappa_{\eta, \phi}(\lambda, \mu)=\varrho^{\lambda, \mu}\left(\omega_{\eta}^{\prime} \omega_{\phi}\right) \quad \text { and } \quad \kappa_{\zeta, \psi}^{i}(\lambda, \mu)=\varrho^{\sigma_{i}(\lambda), \mu}\left(\omega_{\zeta}^{\prime} \omega_{\psi}\right),
$$

then

$$
\sum_{(\eta, \phi)} \theta_{\eta, \phi} \kappa_{\eta, \phi}=\sum_{(\zeta, \psi)} \theta_{\zeta, \psi} \kappa_{\zeta, \psi}^{i} .
$$

Each side of (5.15) is a linear combination of different characters by lemma 5.4. Now, if $\theta_{\eta, \phi} \neq 0$, then $\kappa_{\eta, \phi}=\kappa_{\zeta, \psi}^{i}$ for some $(\zeta, \psi)$. Moreover, for each $1 \leqslant j<n$,

$$
\begin{aligned}
\kappa_{\eta, \phi}\left(0, \varpi_{j}\right) & =\varrho^{0, \varpi_{j}}\left(\omega_{\eta}^{\prime} \omega_{\phi}\right)=\left(r s^{-1}\right)^{\left\langle\eta+\phi, \varpi_{j}\right\rangle} \\
& =\kappa_{\zeta, \psi}^{i}\left(0, \varpi_{j}\right)=\varrho^{0, \varpi_{j}}\left(\omega_{\zeta}^{\prime} \omega_{\psi}\right)=\left(r s^{-1}\right)^{\left\langle\zeta+\psi, \varpi_{j}\right\rangle} .
\end{aligned}
$$


Thus, $\left\langle\eta+\phi, \varpi_{j}\right\rangle=\left\langle\zeta+\psi, \varpi_{j}\right\rangle$ for all $j$, and so

$$
\eta+\phi=\zeta+\psi \text {. }
$$

If $\eta=\sum_{j} \eta_{j} \alpha_{j}, \phi=\sum_{j} \phi_{j} \alpha_{j}, \zeta=\sum_{j} \zeta_{j} \alpha_{j}$ and $\psi=\sum_{j} \psi_{j} \alpha_{j}$, then the equation $\kappa_{\eta, \phi}\left(\varpi_{i}, 0\right)=\kappa_{\zeta, \psi}^{i}\left(\varpi_{i}, 0\right)$ along with $(5.16)$ yields

$$
\eta_{i-1}+\phi_{i-1}+\phi_{i}=\zeta_{i}+\psi_{i-1}+\psi_{i+1} \quad \text { and } \quad \eta_{i-1}+\eta_{i}+\phi_{i-1}=\zeta_{i-1}+\zeta_{i+1}+\psi_{i}
$$

(with the convention that $\eta_{0}=\eta_{n}=\phi_{0}=\phi_{n}=\zeta_{0}=\zeta_{n}=\psi_{0}=\psi_{n}=0$ ). Thus,

$$
\eta_{i-1}+\phi_{i-1}=\eta_{i+1}+\phi_{i+1}, \quad 1 \leqslant i<n .
$$

This implies that if $\theta_{\eta, \phi} \neq 0$, then $\omega_{\eta}^{\prime} \omega_{\phi} \in U_{\natural}^{0}$. As a result, $u \in U_{\natural}^{0}$.

By proposition 5.6, $\varrho^{\lambda, \mu}(u)=\varrho^{\sigma(\lambda), \mu}(u)=\varrho^{\lambda, \mu}\left(\sigma^{-1}(u)\right)$ for all $\lambda, \mu \in \Lambda_{\mathfrak{s l}}$ and $\sigma \in W$. But then $u=\sigma^{-1}(u)$ by corollary 5.5 , so $u \in\left(U_{\natural}^{0}\right)^{W}$, as claimed.

Proposition 5.8. The image of the centre $\mathfrak{Z}$ of $U$ under the Harish-Chandra homomorphism satisfies

$$
\xi(\mathfrak{Z}) \subseteq\left(U_{\natural}^{0}\right)^{W} .
$$

Proof. Assume that $z \in \mathfrak{Z}$. Choose $\mu, \lambda \in \Lambda_{\mathfrak{s l}}$ and assume that $\left\langle\lambda, \alpha_{i}\right\rangle \geqslant 0$ for some (fixed) value $i$. Let $v_{\lambda, \mu} \in M\left(\varrho^{\lambda, \mu}\right)$ be the highest weight vector. Then

$$
z v_{\lambda, \mu}=\pi(z) v_{\lambda, \mu}=\varrho^{\lambda, \mu}(\pi(z)) v_{\lambda, \mu}=\varrho^{\lambda+\rho, \mu}(\xi(z)) v_{\lambda, \mu}
$$

for all $z \in \mathfrak{Z}$. Thus, $z$ acts as the scalar $\varrho^{\lambda+\rho, \mu}(\xi(z))$ on $M\left(\varrho^{\lambda, \mu}\right)$.

Using [5, lemma 2.3], it is easy to see that

$$
e_{i} f_{i}^{\left\langle\lambda, \alpha_{i}\right\rangle+1} v_{\lambda, \mu}=\left(\left[\left\langle\lambda, \alpha_{i}\right\rangle+1\right] f_{i}^{\left\langle\lambda, \alpha_{i}\right\rangle} \frac{r^{-\left\langle\lambda, \alpha_{i}\right\rangle} \omega_{i}-s^{-\left\langle\lambda, \alpha_{i}\right\rangle} \omega_{i}^{\prime}}{r-s}\right) v_{\lambda, \mu}=0,
$$

where, for $k \geqslant 1$,

$$
[k]=\frac{r^{k}-s^{k}}{r-s} .
$$

Thus, $e_{j} f_{i}^{\left\langle\lambda, \alpha_{i}\right\rangle+1} v_{\lambda, \mu}=0$ for all $1 \leqslant j<n$. Note that

$$
\begin{aligned}
z f_{i}^{\left\langle\lambda, \alpha_{i}\right\rangle+1} v_{\lambda, \mu} & =\pi(z) f_{i}^{\left\langle\lambda, \alpha_{i}\right\rangle+1} v_{\lambda, \mu} \\
& =\varrho^{\sigma_{i}(\lambda+\rho)-\rho, \mu}(\pi(z)) f_{i}^{\left\langle\lambda, \alpha_{i}\right\rangle+1} v_{\lambda, \mu} \\
& =\varrho^{\sigma_{i}(\lambda+\rho), \mu}(\xi(z)) f_{i}^{\left\langle\lambda, \alpha_{i}\right\rangle+1} v_{\lambda, \mu} .
\end{aligned}
$$

On the other hand, since $z$ acts as the scalar $\varrho^{\lambda+\rho, \mu}(\xi(z))$ on $M\left(\varrho^{\lambda, \mu}\right)$,

$$
z f_{i}^{\left\langle\lambda, \alpha_{i}\right\rangle+1} v_{\lambda, \mu}=\varrho^{\lambda+\rho, \mu}(\xi(z)) f_{i}^{\left\langle\lambda, \alpha_{i}\right\rangle+1} v_{\lambda, \mu} .
$$

Therefore,

$$
\varrho^{\lambda+\rho, \mu}(\xi(z))=\varrho^{\sigma_{i}(\lambda+\rho), \mu}(\xi(z)) .
$$

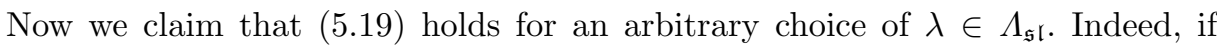
$\left\langle\lambda, \alpha_{i}\right\rangle=-1$, then $\lambda+\rho=\sigma_{i}(\lambda+\rho)$, and so (5.19) holds trivially. For $\lambda$ such that $\left\langle\lambda, \alpha_{i}\right\rangle<-1$, we let $\lambda^{\prime}=\sigma_{i}(\lambda+\rho)-\rho$. Then $\left\langle\lambda^{\prime}, \alpha_{i}\right\rangle \geqslant 0$ and we may apply (5.19) 
to $\lambda^{\prime}$. Substituting $\lambda^{\prime}=\sigma_{i}(\lambda+\rho)-\rho$ into the result, we see that (5.19) holds for this case also.

Since $i$ can be arbitrary, and $W$ is generated by the reflections $\sigma_{i}$, we deduce that

$$
\varrho^{\lambda, \mu}(\xi(z))=\varrho^{\sigma(\lambda), \mu}(\xi(z))
$$

for all $\lambda, \mu \in \Lambda_{\mathfrak{s l}}$ and for all $\sigma \in W$. The assertion of the proposition then follows immediately from lemma 5.7.

Lemma 5.9. $z \in \mathfrak{Z}$ if and only if $\operatorname{ad}(x) z=(\imath \circ \varepsilon)(x) z$ for all $x \in U$, where $\varepsilon: U \rightarrow \mathbb{K}$ is the co-unit and $\imath: \mathbb{K} \rightarrow U$ is the unit of $U$.

Proof. Let $z \in \mathfrak{Z}$. Then, for all $x \in U$,

$$
\operatorname{ad}(x) z=\sum_{(x)} x_{(1)} z S\left(x_{(2)}\right)=z \sum_{(x)} x_{(1)} S\left(x_{(2)}\right)=(\iota \circ \varepsilon)(x) z .
$$

Conversely, assume that $\operatorname{ad}(x) z=(\iota \circ \varepsilon)(x) z$ for all $x \in U$. Then

$$
\omega_{i} z \omega_{i}^{-1}=\operatorname{ad}\left(\omega_{i}\right) z=(\imath \circ \varepsilon)\left(\omega_{i}\right) z=z .
$$

Similarly, $\omega_{i}^{\prime} z\left(\omega_{i}^{\prime}\right)^{-1}=z$. Furthermore,

$$
0=(\imath \circ \varepsilon)\left(e_{i}\right) z=\operatorname{ad}\left(e_{i}\right) z=e_{i} z+\omega_{i} z\left(-\omega_{i}^{-1}\right) e_{i}=e_{i} z-z e_{i}
$$

and

$$
0=(\imath \circ \varepsilon)\left(f_{i}\right) z=\operatorname{ad}\left(f_{i}\right) z=z\left(-f_{i}\left(\omega_{i}^{\prime}\right)^{-1}\right)+f_{i} z\left(\omega_{i}^{\prime}\right)^{-1}=\left(-z f_{i}+f_{i} z\right)\left(\omega_{i}^{\prime}\right)^{-1} .
$$

Hence, $z \in \mathfrak{Z}$.

Lemma 5.10. Assume that $\Psi: U_{-\mu}^{-} \times U_{\nu}^{+} \rightarrow \mathbb{K}$ is a bilinear map, and let $(\eta, \phi) \in$ $Q \times Q$. There then exists $u \in U_{-\nu}^{-} U^{0} U_{\mu}^{+}$such that

$$
\left\langle u \mid\left(y \omega_{\mu}^{\prime-1}\right) \omega_{\eta_{1}}^{\prime} \omega_{\phi_{1}} x\right\rangle=\left(\omega_{\eta_{1}}^{\prime}, \omega_{\phi}\right)\left(\omega_{\eta}^{\prime}, \omega_{\phi_{1}}\right) \Psi(y, x)
$$

for all $x \in U_{\nu}^{+}, y \in U_{-\mu}^{-}$and $\left(\eta_{1}, \phi_{1}\right) \in Q \times Q$.

Proof. As in the proof of proposition 4.11, for each $\mu \in Q^{+}$we choose an arbitrary basis $u_{1}^{\mu}, u_{2}^{\mu}, \ldots, u_{d_{\mu}}^{\mu}\left(d_{\mu}=\operatorname{dim} U_{\mu}^{+}\right)$of $U_{\mu}^{+}$and a dual basis $v_{1}^{\mu}, v_{2}^{\mu}, \ldots, v_{d_{\mu}}^{\mu}$ of $U_{-\mu}^{-}$ such that $\left(v_{i}^{\mu}, u_{j}^{\mu}\right) \stackrel{u_{\mu}}{=} \delta_{i, j}$. If we set

$$
u=\sum_{i, j} \Psi\left(v_{j}^{\mu}, u_{i}^{\nu}\right) v_{i}^{\nu}\left(\omega_{\nu}^{\prime}\right)^{-1} \omega_{\eta}^{\prime} \omega_{\phi} u_{j}^{\mu}\left(r s^{-1}\right)^{-\langle\rho, \nu\rangle},
$$

then it is straightforward to verify that $u$ satisfies equation (5.21).

We define a $U$-module structure on the dual space $U^{*}$ by $(x \cdot f)(v)=f(\operatorname{ad}(S(x)) v)$ for $f \in U^{*}$ and $x \in U$. Also we define a map $\beta: U \rightarrow U^{*}$ by setting

$$
\beta(u)(v)=\langle u \mid v\rangle \text { for } u, v \in U .
$$

Then $\beta$ is an injective $U$-module homomorphism by propositions 4.8 and 4.11 , where the $U$-module structure on $U$ is given by the adjoint action. 
Definition 5.11. Assume that $M$ is a finite-dimensional $U$-module. For each $m \in$ $M$ and $f \in M^{*}$, we define $c_{f, m} \in U^{*}$ by $c_{f, m}(v)=f(v \cdot m), v \in U$.

Proposition 5.12. Assume that $M$ is a finite-dimensional $U$-module such that

$$
M=\bigoplus_{\lambda \in \operatorname{wt}(M)} M_{\lambda} \quad \text { and } \quad \operatorname{wt}(M) \subset Q .
$$

For each $f \in M^{*}$ and $m \in M$, there exists a unique $u \in U$ such that

$$
c_{f, m}(v)=\langle u \mid v\rangle \quad \text { for all } v \in U .
$$

Proof. The uniqueness follows immediately from proposition 4.11. Since $c_{f, m}$ depends linearly on $m$, we may assume that $m \in M_{\lambda}$ for some $\lambda \in Q$. For

$$
v=\left(y \omega_{\mu}^{\prime-1}\right) \omega_{\eta_{1}}^{\prime} \omega_{\phi_{1}} x, \quad x \in U_{\nu}^{+}, \quad y \in U_{-\mu}^{-}, \quad\left(\eta_{1}, \phi_{1}\right) \in Q \times Q,
$$

we have

$$
\begin{aligned}
c_{f, m}(v) & =c_{f, m}\left(\left(y \omega_{\mu}^{\prime-1}\right) \omega_{\eta_{1}}^{\prime} \omega_{\phi_{1}} x\right) \\
& =f\left(\left(y \omega_{\mu}^{\prime-1}\right) \omega_{\eta_{1}}^{\prime} \omega_{\phi_{1}} x m\right) \\
& =\varrho^{\nu+\lambda}\left(\omega_{\eta_{1}}^{\prime} \omega_{\phi_{1}}\right) f\left(\left(y \omega_{\mu}^{\prime-1}\right) x m\right) .
\end{aligned}
$$

Note that $(y, x) \mapsto f\left(\left(y \omega_{\mu}^{\prime-1}\right) x m\right)$ is bilinear, and (4.1) gives us

$$
\left(\omega_{\eta_{1}}^{\prime}, \omega_{-\nu-\lambda}\right)=\varrho^{\nu+\lambda}\left(\omega_{\eta_{1}}^{\prime}\right) \text { and }\left(\omega_{\nu+\lambda}^{\prime}, \omega_{\phi_{1}}\right)=\varrho^{\nu+\lambda}\left(\omega_{\phi_{1}}\right) .
$$

Thus,

$$
c_{f, m}(v)=\left(\omega_{\eta_{1}}^{\prime}, \omega_{-\nu-\lambda}\right)\left(\omega_{\nu+\lambda}^{\prime}, \omega_{\phi_{1}}\right) f\left(y\left(\omega_{\mu}^{\prime}\right)^{-1} x m\right),
$$

and lemma 5.10 enables us to find $u_{\nu \mu} \in U_{-\nu}^{-} U^{0} U_{\mu}^{+}$such that $c_{f, m}(v)=\left\langle u_{\nu \mu} \mid v\right\rangle$ for all $v \in U_{-\mu}^{-} U^{0} U_{\nu}^{+}$.

Now, for an arbitrary $v \in U$, we write $v=\sum_{(\mu, \nu)} v_{\mu \nu}$ with $v_{\mu \nu} \in U_{-\mu}^{-} U^{0} U_{\nu}^{+}$. Since $M$ is finite-dimensional, there is a finite set $\mathcal{F}$ of pairs $(\mu, \nu) \in Q \times Q$ such that

$$
c_{f, m}(v)=c_{f, m}\left(\sum_{(\mu, \nu) \in \mathcal{F}} v_{\mu \nu}\right) \quad \text { for all } v \in U .
$$

Setting $u=\sum_{(\mu, \nu) \in \mathcal{F}} u_{\nu \mu}$ and using lemma 4.7, we have

$$
\begin{aligned}
c_{f, m}(v) & =c_{f, m}\left(\sum_{(\mu, \nu) \in \mathcal{F}} v_{\mu \nu}\right)=\sum_{(\mu, \nu) \in \mathcal{F}} c_{f, m}\left(v_{\mu \nu}\right) \\
& =\sum_{(\mu, \nu) \in \mathcal{F}}\left\langle u_{\nu \mu} \mid v_{\mu \nu}\right\rangle=\sum_{(\mu, \nu) \in \mathcal{F}}\left\langle u_{\nu \mu} \mid v\right\rangle=\langle u \mid v\rangle .
\end{aligned}
$$

This completes the proof.

The category $\mathcal{O}$ of representations of $U$ is naturally defined. We refer the reader to $[4, \S 4]$ for the precise definition. All highest weight modules with weights in $\Lambda_{\mathfrak{s l}}$, such as the Verma modules $M(\lambda)$ and the irreducible modules $L(\lambda)$ for $\lambda \in \Lambda_{\mathfrak{s l}}$, belong to category $\mathcal{O}$. 
Assume that $M$ is any $U$-module in category $\mathcal{O}$, and define a linear map $\Theta$ : $M \rightarrow M$ by

$$
\Theta(m)=\left(r s^{-1}\right)^{-\langle\rho, \lambda\rangle} m
$$

for all $m \in M_{\lambda}, \lambda \in \Lambda_{\mathfrak{s l}}$. We claim that

$$
\Theta u=S^{2}(u) \Theta \text { for all } u \in U .
$$

Indeed, we have only to check this holds when $u$ is one of the generators $e_{i}, f_{i}, \omega_{i}$ or $\omega_{i}^{\prime}$, and for them the verification of (5.24) is straightforward.

For $\lambda \in \Lambda_{\mathfrak{s l}}^{+}$, we define $f_{\lambda} \in U^{*}$ as given by the following trace map:

$$
f_{\lambda}(u)=\operatorname{tr}_{L(\lambda)}(u \Theta), \quad u \in U .
$$

Lemma 5.13. Assume that $\lambda \in \Lambda_{\mathfrak{s l}}^{+} \cap Q$. Then $f_{\lambda} \in \operatorname{Im}(\beta)$, where $\beta$ is defined in equation (5.22).

Proof. Let $k=\operatorname{dim} L(\lambda)$, and fix a basis $\left\{m_{i}\right\}$ for $L(\lambda)$ and its dual basis $\left\{f_{i}\right\}$ for $L(\lambda)^{*}$. We now have

$$
f_{\lambda}(v)=\operatorname{tr}_{L(\lambda)}(v \Theta)=\sum_{i=1}^{k} c_{f_{i}, \Theta m_{i}}(v) .
$$

By proposition 5.12, we can find $u_{i} \in U$ such that $c_{f_{i}, \Theta m_{i}}(v)=\left\langle u_{i} \mid v\right\rangle$ for each $i$, $1 \leqslant i \leqslant k$. Set $u=\sum_{i=1}^{k} u_{i}$ such that

$$
\beta(u)(v)=\sum_{i=1}^{k}\left\langle u_{i} \mid v\right\rangle=\sum_{i=1}^{k} c_{f_{i}, \Theta m_{i}}(v)=f_{\lambda}(v) .
$$

Thus, $f_{\lambda} \in \operatorname{Im}(\beta)$.

Proposition 5.14. The element $z_{\lambda}:=\beta^{-1}\left(f_{\lambda}\right)$ is contained in the centre $\mathfrak{Z}$ for each $\lambda \in \Lambda_{\mathfrak{s l}}^{+} \cap Q$.

Proof. Using (5.24), we have, for all $x \in U$,

$$
\begin{aligned}
\left(S^{-1}(x) f_{\lambda}\right)(u) & =f_{\lambda}(\operatorname{ad}(x) u) \\
& =\operatorname{tr}_{L(\lambda)}\left(\sum_{(x)} x_{(1)} u S\left(x_{(2)}\right) \Theta\right) \\
& =\operatorname{tr}_{L(\lambda)}\left(u \sum_{(x)} S\left(x_{(2)}\right) \Theta x_{(1)}\right) \\
& =\operatorname{tr}_{L(\lambda)}\left(u \sum_{(x)} S\left(x_{(2)}\right) S^{2}\left(x_{(1)}\right) \Theta\right) \\
& =\operatorname{tr}_{L(\lambda)}\left(u S\left(\sum_{(x)} S\left(x_{(1)}\right) x_{(2)}\right) \Theta\right) \\
& =(\imath \circ \varepsilon)(x) \operatorname{tr}_{L(\lambda)}(u \Theta)=(\imath \circ \varepsilon)(x) f_{\lambda}(u) .
\end{aligned}
$$


Substituting $x$ for $S^{-1}(x)$ in the above, we deduce from $\varepsilon \circ S=\varepsilon$ the relation

$$
x f_{\lambda}=(\imath \circ \epsilon)(x) f_{\lambda} .
$$

We can write

$$
x f_{\lambda}=x \beta\left(\beta^{-1}\left(f_{\lambda}\right)\right)=\beta\left(\operatorname{ad}(S(x)) \beta^{-1}\left(f_{\lambda}\right)\right)
$$

and

$$
(\imath \circ \varepsilon)(x) f_{\lambda}=(\imath \circ \varepsilon)(x) \beta\left(\beta^{-1}\left(f_{\lambda}\right)\right)=\beta\left((\imath \circ \varepsilon)(x) \beta^{-1}\left(f_{\lambda}\right)\right) .
$$

Since $\beta$ is injective, $\operatorname{ad}(S(x)) \beta^{-1}\left(f_{\lambda}\right)=(\imath \circ \varepsilon)(x) \beta^{-1}\left(f_{\lambda}\right)$. Since $\varepsilon \circ S^{-1}=\varepsilon$, substituting $x$ for $S(x)$, we obtain

$$
\operatorname{ad}(x) \beta^{-1}\left(f_{\lambda}\right)=(\imath \circ \varepsilon)(x) \beta^{-1}\left(f_{\lambda}\right) \quad \text { for all } x \in U .
$$

Therefore, we may conclude from lemma 5.9 that $\beta^{-1}\left(f_{\lambda}\right) \in \mathfrak{Z}$.

This brings us to our main result on the centre of $U$.

Theorem 5.15. Assume that $r$ and s satisfy condition (5.1).

(i) If $n$ is odd, then the map $\xi: \mathfrak{Z} \rightarrow\left(U_{\natural}^{0}\right)^{W}=\left(U_{b}^{0}\right)^{W}$ is an isomorphism.

(ii) If $n$ is even, the centre $\mathfrak{Z}$ is isomorphic under $\xi$ to a subalgebra of $\left(U_{\natural}^{0}\right)^{W}$ containing $\mathbb{K}\left[\mathfrak{z}, \mathfrak{z}^{-1}\right] \otimes\left(U_{b}^{0}\right)^{W}$, i.e. $\mathbb{K}\left[\mathfrak{z}, \mathfrak{z}^{-1}\right] \otimes\left(U_{b}^{0}\right)^{W} \subseteq \xi(\mathfrak{Z}) \subseteq\left(U_{\mathfrak{b}}^{0}\right)^{W}$, where the element $\mathfrak{z} \in \mathfrak{Z}$ is defined in (5.5).

Proof. We set $z_{\lambda}=\beta^{-1}\left(f_{\lambda}\right)$ for $\lambda \in \Lambda_{\mathfrak{s l}}^{+} \cap Q$ and write

$$
z_{\lambda}=\sum_{\nu \geqslant 0} z_{\lambda, \nu} \quad \text { and } \quad z_{\lambda, 0}=\sum_{(\eta, \phi) \in Q \times Q} \theta_{\eta, \phi} \omega_{\eta}^{\prime} \omega_{\phi}
$$

where $z_{\lambda, \nu} \in U_{-\nu}^{-} U^{0} U_{\nu}^{+}$and $\theta_{\eta, \phi} \in \mathbb{K}$. Then, for $\left(\eta_{1}, \phi_{1}\right) \in Q \times Q$,

$$
\left\langle z_{\lambda} \mid \omega_{\eta_{1}}^{\prime} \omega_{\phi_{1}}\right\rangle=\left\langle z_{\lambda, 0} \mid \omega_{\eta_{1}}^{\prime} \omega_{\phi_{1}}\right\rangle=\sum_{(\eta, \phi)} \theta_{\eta, \phi}\left(\omega_{\eta_{1}}^{\prime}, \omega_{\phi}\right)\left(\omega_{\eta}^{\prime}, \omega_{\phi_{1}}\right) .
$$

On the other hand,

$$
\begin{aligned}
\left\langle z_{\lambda} \mid \omega_{\eta_{1}}^{\prime} \omega_{\phi_{1}}\right\rangle & =\beta\left(z_{\lambda}\right)\left(\omega_{\eta_{1}}^{\prime} \omega_{\phi_{1}}\right)=f_{\lambda}\left(\omega_{\eta_{1}}^{\prime} \omega_{\phi_{1}}\right)=\operatorname{tr}_{L(\lambda)}\left(\omega_{\eta_{1}}^{\prime} \omega_{\phi_{1}} \Theta\right) \\
& =\sum_{\mu \leqslant \lambda} \operatorname{dim}\left(L(\lambda)_{\mu}\right)\left(r s^{-1}\right)^{-\langle\rho, \mu\rangle} \varrho^{\mu}\left(\omega_{\eta_{1}}^{\prime} \omega_{\phi_{1}}\right) \\
& =\sum_{\mu \leqslant \lambda} \operatorname{dim}\left(L(\lambda)_{\mu}\right)\left(r s^{-1}\right)^{-\langle\rho, \mu\rangle}\left(\omega_{\eta_{1}}^{\prime}, \omega_{-\mu}\right)\left(\omega_{\mu}^{\prime}, \omega_{\phi_{1}}\right) .
\end{aligned}
$$

Now we may write

$$
\sum_{(\eta, \phi)} \theta_{\eta, \phi} \chi_{\eta, \phi}=\sum_{\mu \leqslant \nu} \operatorname{dim}\left(L(\lambda)_{\mu}\right)\left(r s^{-1}\right)^{-\langle\rho, \mu\rangle} \chi_{\mu,-\mu},
$$


where the characters $\chi_{\eta, \phi}$ are defined in (4.5). By assumption (5.1), lemma 4.10 and the linear independence of distinct characters, we obtain

$$
\theta_{\eta, \phi}= \begin{cases}\operatorname{dim}\left(L(\lambda)_{\eta}\right)\left(r s^{-1}\right)^{-\langle\rho, \eta\rangle} & \text { if } \eta+\phi=0 \\ 0 & \text { otherwise. }\end{cases}
$$

Hence,

$$
z_{\lambda, 0}=\sum_{\mu \leqslant \lambda} \operatorname{dim}\left(L(\lambda)_{\mu}\right)\left(r s^{-1}\right)^{-\langle\rho, \mu\rangle} \omega_{\mu}^{\prime} \omega_{-\mu}
$$

and, by (5.4),

$$
\xi\left(z_{\lambda}\right)=\varrho^{-\rho}\left(z_{\lambda, 0}\right)=\sum_{\mu \leqslant \lambda} \operatorname{dim}\left(L(\lambda)_{\mu}\right) \omega_{\mu}^{\prime} \omega_{-\mu} .
$$

Note that $\mathfrak{z}=\xi(\mathfrak{z}) \in\left(U_{\mathfrak{\natural}}^{0}\right)^{W}$ when $n$ is even. By propositions 5.2 and 5.8 , it is sufficient to show that $\left(U_{b}^{0}\right)^{W} \subseteq \xi(\mathfrak{Z})$. For $\lambda \in \Lambda_{\mathfrak{s l}}^{+} \cap Q$, we define

$$
\operatorname{av}(\lambda)=\frac{1}{|W|} \sum_{\sigma \in W} \sigma\left(\omega_{\lambda}^{\prime} \omega_{-\lambda}\right)=\frac{1}{|W|} \sum_{\sigma \in W} \omega_{\sigma(\lambda)}^{\prime} \omega_{-\sigma(\lambda)} .
$$

Remembering that, for each $\eta \in Q$, there exists $\sigma \in W$ such that $\sigma(\eta) \in \Lambda_{\mathfrak{s l}}^{+} \cap Q$, we see that the set $\left\{\operatorname{av}(\lambda) \mid \lambda \in \Lambda_{\mathfrak{s l}}^{+} \cap Q\right\}$ forms a basis of $\left(U_{b}^{0}\right)^{W}$. Thus, we have only to show that $\operatorname{av}(\lambda) \in \operatorname{Im}(\xi)$ for all $\lambda \in \Lambda_{\mathfrak{s l}}^{+} \cap Q$. We use induction on $\lambda$. If $\lambda=0, \operatorname{av}(0)=1=\xi(1)$. Assume that $\lambda>0$. Since $\operatorname{dim} L(\lambda)_{\mu}=\operatorname{dim} L(\lambda)_{\sigma(\mu)}$ for all $\sigma \in W$ (proposition 2.3) and $\operatorname{dim} L(\lambda)_{\lambda}=1$, we can rewrite (5.25) to obtain

$$
\xi\left(z_{\lambda}\right)=|W| \operatorname{av}(\lambda)+|W| \sum \operatorname{dim}\left(L(\lambda)_{\mu}\right) \operatorname{av}(\mu),
$$

where the sum is over $\mu$ such that $\mu<\lambda$ and $\mu \in \Lambda_{\mathfrak{s l}}^{+} \cap Q$. By the induction hypothesis, we get $\operatorname{av}(\lambda) \in \operatorname{Im}(\xi)$. This completes the proof.

EXAMPLE 5.16. The centre $\mathfrak{Z}$ of $U=U_{r, s}\left(\mathfrak{s l}_{2}\right)$ has a basis of monomials $\mathfrak{z}^{i} \mathcal{C}^{j}, i \in \mathbb{Z}$, $j \in \mathbb{Z}_{\geqslant 0}$, where $\mathfrak{z}=\omega^{\prime} \omega$ (we omit the subscript since there is only one of them), and $\mathcal{C}$ is the Casimir element,

$$
\mathcal{C}=e f+\frac{s \omega+r \omega^{\prime}}{(r-s)^{2}}=f e+\frac{r \omega+s \omega^{\prime}}{(r-s)^{2}} .
$$

Now

$$
\xi(\mathfrak{z})=\mathfrak{z} \quad \text { and } \quad \xi(\mathcal{C})=\frac{(r s)^{1 / 2}}{(r-s)^{2}}\left(\omega+\omega^{\prime}\right) .
$$

Thus, the monomials $\mathfrak{z}^{i} \mathfrak{c}^{j}, i \in \mathbb{Z}, j \in \mathbb{Z}_{\geqslant 0}$, where $\mathfrak{c}=\omega+\omega^{\prime}$, give a basis for $\xi(\mathfrak{Z})$. The subalgebra $\left(U_{b}^{0}\right)^{W}$ consists of polynomials in $\mathfrak{a}:=\omega^{\prime} \omega^{-1}+\left(\omega^{\prime}\right)^{-1} \omega=2 \operatorname{av}(\alpha)$. Observe that $\mathfrak{a}+2=\mathfrak{z}^{-1} \mathfrak{c}^{2} \in \xi(\mathfrak{Z})$, but we cannot express $\mathfrak{c}$ as an element of $\mathbb{K}\left[\mathfrak{z}, \mathfrak{z}^{-1}\right] \otimes\left(U_{b}^{0}\right)^{W}$. Since $\sigma\left(\left(\omega^{\prime}\right)^{\ell} \omega^{m}\right)=\left(\omega^{\prime}\right)^{m} \omega^{\ell}$, we see that $\left(U^{0}\right)^{W}$ has as a basis the sums $\left(\omega^{\prime}\right)^{\ell} \omega^{m}+\left(\omega^{\prime}\right)^{m} \omega^{\ell}$ for all $\ell, m \in \mathbb{Z}$, and hence $\mathbb{K}\left[\mathfrak{z}, \mathfrak{z}^{-1}\right] \otimes\left(U_{b}^{0}\right)^{W} \subsetneq \xi(\mathfrak{Z})=$ $\left(U_{\natural}^{0}\right)^{W}=\left(U^{0}\right)^{W}$, as no conditions are imposed by $(5.9)$. 


\section{Acknowledgments}

The authors thank the referee for many helpful suggestions. G.B. was supported by NSF Grant no. DMS-0245082, NSA Grant no. MDA904-03-1-0068, and gratefully acknowledges the hospitality of Seoul National University. S.-J.K. was supported in part by KOSEF Grant no. R01-2003-000-10012-0 and KRF Grant no. 2003-070C00001. K.-H.L. was also supported in part by KOSEF Grant no. R01-2003-00010012-0.

\section{Appendix A.}

Lemma A.1. The relations

(i) $\mathcal{E}_{i, j} \mathcal{E}_{k, l}-\mathcal{E}_{k, l} \mathcal{E}_{i, j}=0$, for $i \geqslant j>k+1 \geqslant l+1$,

(ii) $\mathcal{E}_{i, j} \mathcal{E}_{k, l}-r^{-1} \mathcal{E}_{k, l} \mathcal{E}_{i, j}-\mathcal{E}_{i, l}=0$, for $i \geqslant j=k+1 \geqslant l+1$,

(iii) $\mathcal{E}_{i, j} e_{j}-s^{-1} e_{j} \mathcal{E}_{i, j}=0$, for $i>j$,

hold in $U^{+}$.

Proof. The equations in (i) are obvious.

For (ii), we fix $j$ and $l$ with $j>l$ and use induction on $i$. If $i=j$, this is just the definition of $\mathcal{E}_{i, l}$ from (3.1). Assume that $i>j$. We then have

$$
\begin{aligned}
\mathcal{E}_{i, j} \mathcal{E}_{j-1, l} & =e_{i} \mathcal{E}_{i-1, j} \mathcal{E}_{j-1, l}-r^{-1} \mathcal{E}_{i-1, j} e_{i} \mathcal{E}_{j-1, l} \\
& =r^{-1} e_{i} \mathcal{E}_{j-1, l} \mathcal{E}_{i-1, j}+e_{i} \mathcal{E}_{i-1, l}-r^{-2} \mathcal{E}_{j-1, l} \mathcal{E}_{i-1, j} e_{i}-r^{-1} \mathcal{E}_{i-1, l} e_{i} \\
& =r^{-1} \mathcal{E}_{j-1, l} \mathcal{E}_{i, j}+\mathcal{E}_{i, l}
\end{aligned}
$$

by part (i) and the induction hypothesis.

To establish (iii), we fix $j$ and use induction on $i$. When $i=j+1$, the relation is simply (3.2) with $j$ instead of $i$. Assume that $i>j+1$. We then have

$$
\begin{aligned}
\mathcal{E}_{i, j} e_{j} & =e_{i} \mathcal{E}_{i-1, j} e_{j}-r^{-1} \mathcal{E}_{i-1, j} e_{j} e_{i} \\
& =s^{-1} e_{j} e_{i} \mathcal{E}_{i-1, j}-r^{-1} s^{-1} e_{j} \mathcal{E}_{i-1, j} e_{i} \\
& =s^{-1} e_{j} \mathcal{E}_{i, j}
\end{aligned}
$$

by (i) and induction.

Lemma A.2. In $U^{+}$,

(i) $\mathcal{E}_{i, j} \mathcal{E}_{j, l}-r^{-1} s^{-1} \mathcal{E}_{j, l} \mathcal{E}_{i, j}+\left(r^{-1}-s^{-1}\right) e_{j} \mathcal{E}_{i, l}=0$, for $i>j>l$,

(ii) $\mathcal{E}_{i, j} \mathcal{E}_{k, l}-\mathcal{E}_{k, l} \mathcal{E}_{i, j}=0$, for $i>k \geqslant l>j$.

Proof. The following expression can be easily verified by induction on $l$ :

$$
\mathcal{E}_{i, j} \mathcal{E}_{j, l}-r^{-1} s^{-1} \mathcal{E}_{j, l} \mathcal{E}_{i, j}+r^{-1} \mathcal{E}_{i, l} e_{j}-s^{-1} e_{j} \mathcal{E}_{i, l}=0, \quad i>j>l .
$$

We claim that

$$
\mathcal{E}_{j+1, j-1} e_{j}-e_{j} \mathcal{E}_{j+1, j-1}=0 .
$$


Indeed, we have $e_{j} \mathcal{E}_{j, j-1}=s^{-1} \mathcal{E}_{j, j-1} e_{j}$ as in (3.3), and using this we get

$$
\begin{aligned}
\mathcal{E}_{j+1, j} & \mathcal{E}_{j, j-1}-r^{-1} s^{-1} \mathcal{E}_{j, j-1} \mathcal{E}_{j+1, j} \\
& =e_{j+1} e_{j} \mathcal{E}_{j, j-1}-r^{-1} e_{j} e_{j+1} \mathcal{E}_{j, j-1}-r^{-1} s^{-1} \mathcal{E}_{j, j-1} e_{j+1} e_{j}+r^{-2} s^{-1} \mathcal{E}_{j, j-1} e_{j} e_{j+1} \\
& =s^{-1} e_{j+1} \mathcal{E}_{j, j-1} e_{j}-r^{-1} e_{j} e_{j+1} \mathcal{E}_{j, j-1}-r^{-1} s^{-1} \mathcal{E}_{j, j-1} e_{j+1} e_{j}+r^{-2} e_{j} \mathcal{E}_{j, j-1} e_{j+1} \\
& =s^{-1} \mathcal{E}_{j+1, j-1} e_{j}-r^{-1} e_{j} \mathcal{E}_{j+1, j-1} .
\end{aligned}
$$

On the other hand, we also have, from (A 1),

$$
\mathcal{E}_{j+1, j} \mathcal{E}_{j, j-1}-r^{-1} s^{-1} \mathcal{E}_{j, j-1} \mathcal{E}_{j+1, j}=s^{-1} e_{j} \mathcal{E}_{j+1, j-1}-r^{-1} \mathcal{E}_{j+1, j-1} e_{j}
$$

such that

$$
\left(r^{-1}+s^{-1}\right) \mathcal{E}_{j+1, j-1} e_{j}-\left(r^{-1}+s^{-1}\right) e_{j} \mathcal{E}_{j+1, j-1}=0
$$

Since we have assumed that $r^{-1}+s^{-1} \neq 0$, this implies (A 2).

Now to demonstrate that

$$
\mathcal{E}_{i, j} e_{k}-e_{k} \mathcal{E}_{i, j}=0, \quad i>k>j,
$$

we fix $k$, and assume first that $j=k-1$. The argument proceeds by induction on $i$. If $i=k+1$, then the expression in (A 3) becomes (A 2) (with $k$ instead of $j$ there). When $i>k+1$,

$$
\begin{aligned}
\mathcal{E}_{i, k-1} e_{k} & =e_{i} \mathcal{E}_{i-1, k-1} e_{k}-r^{-1} \mathcal{E}_{i-1, k-1} e_{k} e_{i} \\
& =e_{k} e_{i} \mathcal{E}_{i-1, k-1}-r^{-1} e_{k} \mathcal{E}_{i-1, k-1} e_{i}=e_{k} \mathcal{E}_{i, k-1}
\end{aligned}
$$

For the case $j<k-1$, we have by induction on $j$,

$$
\begin{aligned}
\mathcal{E}_{i, j} e_{k} & =\mathcal{E}_{i, j+1} e_{j} e_{k}-r^{-1} e_{j} \mathcal{E}_{i, j+1} e_{k} \\
& =e_{k} \mathcal{E}_{i, j+1} e_{j}-r^{-1} e_{k} e_{j} \mathcal{E}_{i, j+1} \\
& =e_{k} \mathcal{E}_{i, j}
\end{aligned}
$$

so that (A 3) is verified.

As a consequence, the relations in part (i) follow from (A 1) and (A 3), while those in (ii) can be derived easily from (A 3) by fixing $i, j$ and $k$ and using induction on $l$.

LEMMA A.3. The relations

(i) $\mathcal{E}_{i, j} \mathcal{E}_{k, j}-s^{-1} \mathcal{E}_{k, j} \mathcal{E}_{i, j}=0$, for $i>k>j$,

(ii) $\mathcal{E}_{i, j} \mathcal{E}_{k, l}-r^{-1} s^{-1} \mathcal{E}_{k, l} \mathcal{E}_{i, j}+\left(r^{-1}-s^{-1}\right) \mathcal{E}_{k, j} \mathcal{E}_{i, l}=0$ for $i>k>j>l$, hold in $U^{+}$. 
Proof. Part (i) follows from lemmas A.1(iii) and A.2(ii). For (ii), we apply induction on $l$. When $l=j-1$, part (i), and lemmas A.1(ii) and A.2(ii) imply that

$$
\begin{aligned}
\mathcal{E}_{i, j} & \mathcal{E}_{k, j-1} \\
& =\mathcal{E}_{i, j} \mathcal{E}_{k, j} e_{j-1}-r^{-1} \mathcal{E}_{i, j} e_{j-1} \mathcal{E}_{k, j} \\
& =s^{-1} \mathcal{E}_{k, j} \mathcal{E}_{i, j} e_{j-1}-r^{-1} \mathcal{E}_{i, j} e_{j-1} \mathcal{E}_{k, j} \\
& =r^{-1} s^{-1} \mathcal{E}_{k, j} e_{j-1} \mathcal{E}_{i, j}+s^{-1} \mathcal{E}_{k, j} \mathcal{E}_{i, j-1}-r^{-2} e_{j-1} \mathcal{E}_{i, j} \mathcal{E}_{k, j}-r^{-1} \mathcal{E}_{i, j-1} \mathcal{E}_{k, j} \\
& =r^{-1} s^{-1} \mathcal{E}_{k, j} e_{j-1} \mathcal{E}_{i, j}+s^{-1} \mathcal{E}_{k, j} \mathcal{E}_{i, j-1}-r^{-2} s^{-1} e_{j-1} \mathcal{E}_{k, j} \mathcal{E}_{i, j}-r^{-1} \mathcal{E}_{k, j} \mathcal{E}_{i, j-1} \\
& =r^{-1} s^{-1} \mathcal{E}_{k, j-1} \mathcal{E}_{i, j}+\left(s^{-1}-r^{-1}\right) \mathcal{E}_{k, j} \mathcal{E}_{i, j-1} .
\end{aligned}
$$

Now assume that $l<j-1$. Then $\mathcal{E}_{i, j} e_{l}=e_{l} \mathcal{E}_{i, j}$ and $\mathcal{E}_{k, j} e_{l}=e_{l} \mathcal{E}_{k, j}$ by lemma A.1(i) and so, by lemma A.1(ii), we obtain

$$
\begin{aligned}
\mathcal{E}_{i, j} \mathcal{E}_{k, l}= & \mathcal{E}_{i, j} \mathcal{E}_{k, l+1} e_{l}-r^{-1} \mathcal{E}_{i, j} e_{l} \mathcal{E}_{k, l+1} \\
= & r^{-1} s^{-1} \mathcal{E}_{k, l+1} e_{l} \mathcal{E}_{i, j}+\left(s^{-1}-r^{-1}\right) \mathcal{E}_{k, j} \mathcal{E}_{i, l+1} e_{l} \\
& \quad-r^{-2} s^{-1} e_{l} \mathcal{E}_{k, l+1} \mathcal{E}_{i, j}-r^{-1}\left(s^{-1}-r^{-1}\right) e_{l} \mathcal{E}_{k, j} \mathcal{E}_{i, l+1} \\
= & r^{-1} s^{-1} \mathcal{E}_{k, l} \mathcal{E}_{i, j}+\left(s^{-1}-r^{-1}\right) \mathcal{E}_{k, j} \mathcal{E}_{i, l}
\end{aligned}
$$

by the induction assumption.

LEMMA A.4. In $U^{+}$,

$$
\mathcal{E}_{i, j} \mathcal{E}_{i, l}-s^{-1} \mathcal{E}_{i, l} \mathcal{E}_{i, j}=0, \quad i \geqslant j>l .
$$

Proof. First consider the case $i=j$. If $l=i-1$, the above relation is merely the defining relation in (3.3). Assume that $l<i-1$. By induction on $l$, we have

$$
\begin{aligned}
e_{i} \mathcal{E}_{i, l} & =e_{i} \mathcal{E}_{i, l+1} e_{l}-r^{-1} e_{i} e_{l} \mathcal{E}_{i, l+1} \\
& =s^{-1} \mathcal{E}_{i, l+1} e_{l} e_{i}-r^{-1} s^{-1} e_{l} \mathcal{E}_{i, l+1} e_{i} \\
& =s^{-1} \mathcal{E}_{i, l} e_{i} .
\end{aligned}
$$

When $i>j$, by induction on $j$ and lemma A.2(ii), we get

$$
\begin{aligned}
\mathcal{E}_{i, j} \mathcal{E}_{i, l} & =\mathcal{E}_{i, j+1} e_{j} \mathcal{E}_{i, l}-r^{-1} e_{j} \mathcal{E}_{i, j+1} \mathcal{E}_{i, l} \\
& =\mathcal{E}_{i, j+1} \mathcal{E}_{i, l} e_{j}-r^{-1} s^{-1} e_{j} \mathcal{E}_{i, l} \mathcal{E}_{i, j+1}, \\
& =s^{-1} \mathcal{E}_{i, l} \mathcal{E}_{i, j+1} e_{j}-r^{-1} s^{-1} \mathcal{E}_{i, l} e_{j} \mathcal{E}_{i, j+1} \\
& =s^{-1} \mathcal{E}_{i, l} \mathcal{E}_{i, j} .
\end{aligned}
$$

The proof of theorem 3.1 is now complete because we have

(1) $\Longleftrightarrow$ lemma A.1(ii);

(2) $\Longleftrightarrow$ lemma A.1(i) and lemma A.2(ii);

$(3) \Longleftrightarrow$ lemma A.1(iii), lemma A.3(i), and lemma A.4;

(4) $\Longleftrightarrow$ lemma A.2(i) and lemma A.3(ii). 


\section{References}

1 P. Baumann. On the center of quantized enveloping algebras. J. Alg. 203 (1998), 244-260.

2 G. Benkart and T. Roby. Down-up algebras. J. Alg. 209 (1998), 305-344. (Addendum 213 (1999), 378.)

3 G. Benkart and S. Witherspoon. A Hopf structure for down-up algebras. Math. Z. 238 (2001), 523-553.

4 G. Benkart and S. Witherspoon. Two-parameter quantum groups and Drinfel'd doubles. Alg. Representat. Theory 7 (2004), 261-286.

5 G. Benkart and S. Witherspoon. Representations of two-parameter quantum groups and Schur-Weyl duality. In Hopf algebras (ed. J. Bergen, S. Catoiu and W. Chin). Lecture Notes in Pure and Applied Mathematics, vol. 237, pp. 62-95. (New York: Marcel Dekker, 2004).

6 G. Benkart and S. Witherspoon. Restricted two-parameter quantum groups. In Finite dimensional algebras and related topics, Fields Institute Communications, vol. 40, pp. 293318 (Providence, RI: American Mathematical Society, 2004).

L. A. Bokut and P. Malcolmson. Gröbner-Shirshov bases for quantum enveloping algebras. Israel J. Math. 96 (1996), 97-113.

8 W. Chin and I. M. Musson. Multiparameter quantum enveloping algebras. J. Pure Appl. Alg. 107 (1996), 171-191.

9 R. Dipper and S. Donkin. Quantum GL . Proc. Lond. Math. Soc. 63 (1991), 165-211.

10 V. K. Dobrev and P. Parashar. Duality for multiparametric quantum $G L(n)$. J. Phys. A 26 (1993), 6991-7002.

11 V. G. Drinfel'd. Quantum groups. In Proc. Int. Cong. of Mathematicians, Berkeley, 1986 (ed. A. M. Gleason), pp. 798-820 (Providence, RI: American Mathematical Society, 1987). V. G. Drinfel'd. Almost cocommutative Hopf algebras. Leningrad Math. J. 1 (1990), 321342.

13 P. I. Etingof. Central elements for quantum affine algebras and affine Macdonald's operators. Math. Res. Lett. 2 (1995), 611-628.

14 K. R. Goodearl and E. S. Letzter. Prime factor algebras of the coordinate ring of quantum matrices. Proc. Am. Math. Soc. 121 (1994), 1017-1025.

15 J. A. Green. Hall algebras, hereditary algebras and quantum groups. Invent. Math. 120 (1995), 361-377.

16 J. Hong. Center and universal $R$-matrix for quantized Borcherds superalgebras. J. Math. Phys. 40 (1999), 3123-3145.

17 N. Jacobson. Basic algebra, vol. 1 (New York: Freeman, 1989).

18 A. Joseph. Quantum groups and their primitive ideals. Ergebnisse der Mathematik und ihrer Grenzgebiete, series 3, vol. 29 (Springer, 1995).

19 A. Joseph, and G. Letzter. Separation of variables for quantized enveloping algebras. Am. J. Math. 116 (1994), 127-177.

20 S.-J. Kang and T. Tanisaki. Universal $R$-matrices and the center of the quantum generalized Kac-Moody algebras. Hiroshima Math. J. 27 (1997), 347-360.

21 V. K. Kharchenko. A combinatorial approach to the quantification of Lie algebras. Pac. J. Math. 203 (2002), 191-233.

22 S. M. Khoroshkin and V. N. Tolstoy. Universal $R$-matrix for quantized (super)algebras. Lett. Math. Phys. 10 (1985), 63-69.

23 S. M. Khoroshkin and V. N. Tolstoy. The Cartan-Weyl basis and the universal $R$-matrix for quantum Kac-Moody algebras and superalgebras. In Quantum Symmetries, Proc. Int. Symp. on Mathematical Physics, Goslar, 1991, pp. 336-351 (River Edge, NJ: World Scientific, 1993).

24 S. M. Khoroshkin and V. N. Tolstoy. Twisting of quantum (super)algebras. In Generalized Symmetries in Physics, Proc. Int. Symp. on Mathematical Physics, Clausthal, 1993, pp. 42-54 (River Edge, NJ: World Scientific, 1994).

S. Levendorskii and Y. Soibelman. Algebras of functions of compact quantum groups, Schubert cells and quantum tori. Commun. Math. Phys. 139 (1991), 141-170.

26 G. Lusztig. Finite dimensional Hopf algebras arising from quantum groups. J. Am. Math. Soc. 3 (1990), 257-296. 
27 G. Lusztig. Canonical bases arising from quantized enveloping algebras. J. Am. Math. Soc. 3 (1990), 447-498.

28 N. Yu. Reshetikhin. Quasitriangular Hopf algebras and invariants of links. Leningrad Math. J. 1 (1990), 491-513.

29 N. Yu. Reshetikhin, L. A. Takhtadzhyan and L. D. Faddeev. Quantization of Lie groups and Lie algebras. Leningrad Math. J. 1 (1990), 193-225.

30 C. M. Ringel. Hall algebras. Banach Center Publicat. 26 (1990), 433-447.

31 C. M. Ringel. Hall algebras and quantum groups. Invent. Math. 101 (1990), 583-591.

32 C. M. Ringel. Hall algebras revisited. Israel Math. Conf. Proc. 7 (1993), 171-176.

C. M. Ringel. PBW-bases of quantum groups. J. Reine Angew. Math. 470 (1996), 51-88.

34 M. Rosso. Analogues de la forme de Killing et du théorème d'Harish-Chandra pour les groupes quantiques. Annls Scient. Éc. Norm. Sup. 23 (1990), 445-467.

M. Takeuchi. A two-parameter quantization of $G L(n)$. Proc. Jpn Acad. A 66 (1990), 112114.

M. Takeuchi. The $q$-bracket product and quantum enveloping algebras of classical types. $J$. Math. Soc. Jpn 42 (1990), 605-629.

T. Tanisaki. Killing forms, Harish-Chandra isomorphisms, and universal $R$-matrices for quantum algebras. Int. J. Mod. Phys. A 7 (suppl. 01B) (1992), 941-961.

N. Xi. Root vectors in quantum groups. Comment. Math. Helv. 69 (1994), 612-639.

39 H. Yamane. A Poincaré-Birkhoff-Witt theorem for the quantum group of type $A_{N}$. Proc. Jpn Acad. A 64 (1988), 385-386.

40 H. Yamane. A Poincaré-Birkhoff-Witt theorem for quantized universal enveloping algebras of type $A_{N}$. Publ. RIMS Kyoto 25 (1989), 503-520.

(Issued 9 June 2006) 